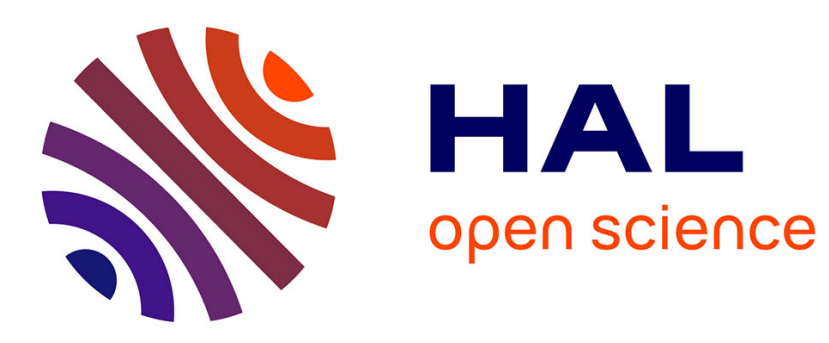

\title{
Role of discrete intra-granular slip bands on the strain-hardening of polycrystals
}

Christophe Collard, Véronique Favier, Marcel Berveiller, Stephane Berbenni

\section{To cite this version:}

Christophe Collard, Véronique Favier, Marcel Berveiller, Stephane Berbenni. Role of discrete intragranular slip bands on the strain-hardening of polycrystals. International Journal of Plasticity, 2010, 26 (2), pp.310-328. 10.1016/j.ijplas.2009.07.004 . hal-02456094

\section{HAL Id: hal-02456094 \\ https://hal.science/hal-02456094}

Submitted on 27 Jan 2020

HAL is a multi-disciplinary open access archive for the deposit and dissemination of scientific research documents, whether they are published or not. The documents may come from teaching and research institutions in France or abroad, or from public or private research centers.
L'archive ouverte pluridisciplinaire HAL, est destinée au dépôt et à la diffusion de documents scientifiques de niveau recherche, publiés ou non, émanant des établissements d'enseignement et de recherche français ou étrangers, des laboratoires publics ou privés. 


\title{
Role of discrete intra-granular slip bands on the strain-hardening of polycrystals
}

\author{
Christophe Collard ${ }^{\mathrm{a}}$, Véronique Favier ${ }^{\mathrm{b}}$, Stéphane Berbenni ${ }^{\mathrm{a}, *}$, Marcel Berveiller ${ }^{\mathrm{a}}$ \\ ${ }^{\text {a }}$ Laboratoire de Physique et Mécanique des Matériaux, LPMM, CNRS, Arts et Metiers ParisTech, 57078 Metz, France \\ ${ }^{\mathrm{b}}$ Laboratoire d'Ingénierie des Matériaux, LIM, CNRS, Arts et Metiers ParisTech, 75013 Paris, France
}

Keywords:

Inelastic deformation

Internal lengths

Discrete slip bands

Micromechanics

Polycrystals

\begin{abstract}
A B S T R A C T
This work investigates a new micromechanical modeling of polycrystal plasticity, accounting slip bands for physical plastic heterogeneities considered as periodically distributed within grains. These intra-granular plastic heterogeneities are modeled by parallel flat ellipsoidal sub-domains, each of them may have a distinct uniform plastic slip. To capture the morphology of slip bands occurring in plastically deforming polycrystals, these interacting sub-domains are considered as oblate spheroids periodically distributed and constrained by spherical grain boundaries. In this paper, we focus the study on the influences of internal length scale parameters related to grain size, spatial period and thickness of slip bands on the overall material's behavior. In a first part, the Gibbs free energy accounting for elastic interactions between plastic heterogeneities is calculated thanks to the Green function's method in the case of an isolated spherical grain with plastic strain occurring only in slip bands embedded in an infinite elastic matrix. In a second part, the influence of discrete periodic distributions of intra-granular slip bands on the polycrystal's behavior is investigated considering an aggregate with random crystallographic orientations. When the spatial period of slip bands is on the same order as the grain radius, the polycrystal's mechanical behavior is found strongly dependent on the ratio between the spatial period of slip bands and the grain size, as well as the ratio between the slip band thickness and the grain size, which cannot be captured by classic length scale independent Eshelby-based micromechanics.
\end{abstract}

\section{Introduction}

The spatial heterogeneity of plastic flow was experimentally highlighted by Neuhäuser (1983). The observation of surface reliefs of metals revealed that slip consists of discrete events localized along slip bands. The slip patterns emerging at the surface can be observed during tensile or compression tests on single and polycrystals with large as well as fine grains for a variety of metals using experimental techniques such as optical micrography, EBSD or AFM as reported by Hirth (1972); Margolin and Stanescu (1975); Villechaise et al. (2002) and Fréchard et al. (2006). From Neuhäuser (1983), observed slip line patterns (e.g. in pure fcc crystals) are more or less "homogeneous" after the onset of plasticity (stage I for single crystals) and gradually slip bands occur (under a more localized plastic deformation especially from the stage II of single crystals).

Scale transition models for polycrystals, such as classic self-consistent schemes for linear or non-linear behaviors (Hill, 1965; Hutchinson, 1976; Berveiller and Zaoui, 1979; Weng, 1980; Molinari et al., 1987; Lebensohn and Tomé, 1993;

\footnotetext{
* Corresponding author. Tel.: +33 35500 4034; fax: +33 387374284 .

E-mail address: Stephane.Berbenni@metz.ensam.fr (S. Berbenni).
} 
Gilormini, 1995; Li and Weng, 1997; Masson et al., 2000; Paquin et al., 2001; Sabar et al., 2002; Molinari, 2002; Berbenni et al., 2004; Pierard and Doghri, 2006; Berbenni et al., 2007; Mercier and Molinari, 2009), are based on a good description of both the grain behavior and the mechanical interactions between grains. These models are successful to describe macroscopic stressstrain responses, internal stresses, crystallographic texture evolution under different loading paths, but do not capture internal length effects inherent to the induced intra-granular microstructure, especially its influence on the grain size effect (Armstrong et al., 1962). This crucial shortcoming is due, at least partly, to the fact that a mean-field approximation neglects the strong inhomogeneity of intra-granular plastic slip and especially its discrete nature under slip bands constrained by grain boundaries.

Other modeling techniques like full discrete dislocation dynamics simulations (Kubin et al., 1992; VanderGiessen and Needleman, 1995; Verdier et al., 1998; Schwarz, 1999) are currently achieved to predict internal length effects (Cleveringa et al., 1998; Groh et al., 2005; Espinosa et al., 2005; Lefebvre et al., 2007; Balint et al., 2008) but the simulations become quickly time consuming for polycrystals. Generalized continuum theories are more tractable for engineering applications but need the introduction of new degrees of freedom like in strain gradient plasticity (Aifantis, 1987; Fleck and Hutchinson, 1997; Acharya and Bassani, 2000; Cheong et al., 2005; Aifantis and Willis, 2005) or in Cosserat media (Forest et al., 2000; Forest and Sedlacek, 2003). Another interesting investigation developed by Acharya and co-workers (Acharya, 2001; Acharya and Roy, 2006) is a continuum dislocation field theory based on non-linear dislocation transport which accounts for phenomenologically short range dislocation interactions. Using a statistical mechanics framework, Groma et al. (2003) and Zaiser and Aifantis (2006) predicted a size-dependent local back-stress but took partly into account the short range interactions between dislocations through spatial correlations as indicated by Roy et al. (2008).

In a previous paper (Berbenni et al., 2008), intra-granular plastic slip heterogeneities have been first modeled by periodic distributions of circular glide dislocation loops for a grain embedded in an infinite elastic matrix. The mechanical fields as well as the elastic energy have been computed using a Fourier Transform method. A strong discrepancy of internal stresses with the classic solution (Eshelby, 1957) has been found especially in a region close to the grain boundary. Furthermore, an internal length effect has been reported regarding the elastic energy which scales with the inverse of the grain size when the spatial period of loops is kept constant. Even though this modeling accounts for the discrete nature of slip through the Burgers vectors and distributions of equally-spaced loops, the number of loops to describe slip bands may become prohibitive. In the present study, slip bands are modeled by oblate spheroids with uniform (i.e. averaged) plastic slip inside them. Thus, compared to discrete distributions of dislocation loops, each oblate spheroid may represent numerous dislocations by averaging their Burgers vectors. In addition, their thickness constitutes a new internal length parameter. In this paper, we determine how internal lengths, related to the initial granular microstructure and the induced intra-granular discrete slips, play a key role on the overall mechanical behavior of polycrystals. For this purpose, we develop a new micromechanical approach accounting for discrete periodic slip band distribution. Periodic configurations (Fig. 1) appear to be the simplest way as a starting point to explore the impact of the induced intra-granular microstructure on the strain-hardening of polycrystals. The plastic deformation is thus characterized by three physical internal parameters: (i) the spatial period of slip bands (denoted $h$ ), (ii) the thickness of the bands (denoted $c$ ), and, (iii) the slip amplitudes inside bands.

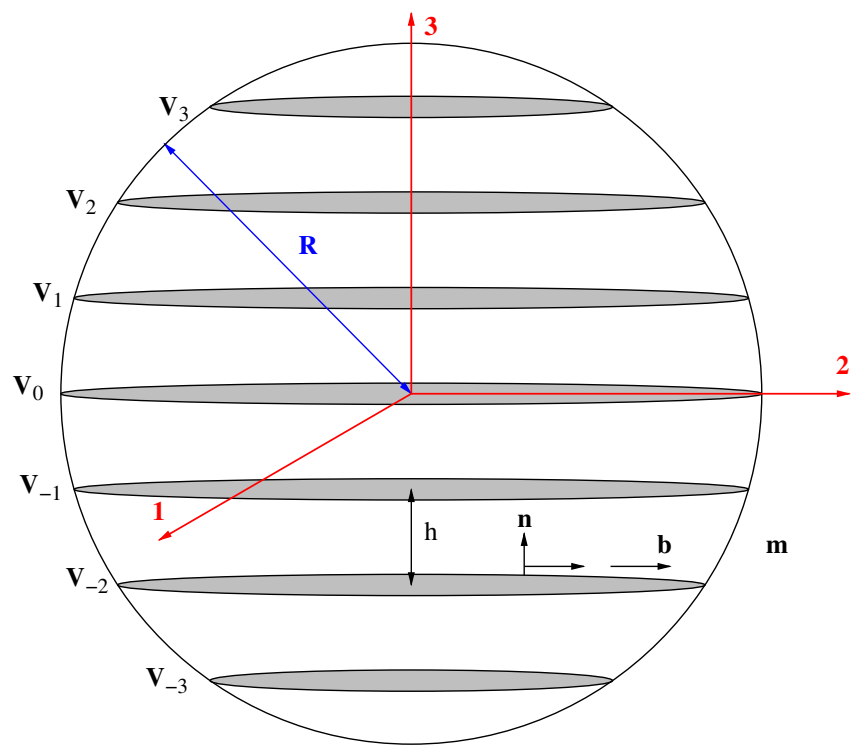

Fig. 1. Distribution of slip bands with individual volumes $V_{I}$ and spatial period $h$ constrained by a spherical grain with radius $R$. The plastic strain in each band is due to slip in the direction of Burgers vector $\mathbf{b}$ in the single slip plane of unit normal $\mathbf{n}$. The grain is embedded in an infinite elastic matrix. 
In Section 2, we present the general theory based on the Green's function method by starting from the field equations and the thermodynamics related to a single grain containing periodic distributions of slip bands embedded in an infinite elastic matrix. Numerical results are presented on the influences of non-dimensional internal lengths (i.e. the ratios of slip band spacing and thickness with grain size) on internal Gibbs free energy. In Section 3, the case of polycrystalline aggregates containing grains only deforming in single slip in the form of slip bands is investigated. To retrieve the macroscopic behavior of the polycrystal, a simple scale transition scheme based on the diluted Eshelby's formalism is used in a first attempt, since we only consider the initial stage of deformation. Let us note that in this paper, we do not focus the study on a homogenization problem but on the influence of the collective behavior of defects (dislocations) which can be represented by intra-granular (plastic) slip bands in an elastic continuum. The results focus on the strain-hardening of polycrystals with random crystallographic orientations during uniaxial tensile deformation. The stress-strain curves for various periodic distributions of intra-granular slip bands (i.e. $\frac{h}{R}$ ) and slip band thicknesses (i.e. $\frac{c}{R}$ ) are analyzed. We find that accounting for the discrete distribution of plastic deformation results in an additional strain hardening with respect to the classic Eshelby's formalism. Moreover, non-dimensional internal parameters of the microstructure $\left(\frac{h}{R}\right.$ and $\left.\frac{c}{R}\right)$ appear to have strong influences on the strain-hardening of polycrystals when the spatial period of slip bands is on the same order as the grain radius. For such slip band configurations, an increase of $\frac{h}{R}$, or, a decrease of $\frac{c}{R}$, leads to an increase of the polycrystal's flow stress.

In the whole paper, we use the following conventions: Latin indices take their values in the set $\{1,2,3\}$, and the Einstein summation convention is used. Bold symbols indicate either tensors or vectors. We also use ":" for contracted product between tensors and "." for simple product.

\section{Discrete distribution of intra-granular plastic heterogeneities: fields equations and applications}

Fields equations and thermodynamics are used here to calculate the Gibbs free energy $\psi$ and the internal stress field $\tau$ of static configurations of slip heterogeneities in an infinite elastic medium related to a single grain. The discrepancies with the classic Eshelby's inclusion scheme are highlighted.

\subsection{Field equations and thermodynamics}

We consider a spherical inclusion with volume $V_{g}$ embedded in an infinite medium denoted $V\left(V \gg V_{g}\right)$. The matrix surrounding the inclusion is assumed to be purely elastic whereas inside the inclusion a plastic strain field is prescribed. We impose on the boundary $\partial V$ of the medium surface tractions $\mathbf{t}^{d}=\boldsymbol{\Sigma} . \mathbf{n}$ and we assume that there is no volume force. Under these conditions, the medium undergoes a displacement field $\mathbf{u}(r)$, related to the strain tensor $\boldsymbol{\varepsilon}(r)$ in the small perturbation hypothesis by:

$$
\boldsymbol{\varepsilon}(r)=\frac{1}{2}\left(\nabla \mathbf{u}(r)+{ }^{t} \nabla \mathbf{u}(r)\right)
$$

This compatible strain splits into an elastic strain $\boldsymbol{\varepsilon}^{e}(r)$ and a plastic strain $\boldsymbol{\varepsilon}^{p}(r)$ so that $\boldsymbol{\varepsilon}(r)=\boldsymbol{\varepsilon}^{e}(r)+\boldsymbol{\varepsilon}^{p}(r)$. The Cauchy stress field $\sigma(r)$ satisfies:

$$
\left\{\begin{array}{l}
\operatorname{div} \boldsymbol{\sigma}(r)=0 \text { in } V \\
\mathbf{t}^{d}=\boldsymbol{\sigma}(r) \cdot \mathbf{n}(r)=\boldsymbol{\Sigma} \cdot \mathbf{n}(r) \text { on } \partial V
\end{array}\right.
$$

The linear and homogeneous elastic behavior is described by the Hooke's law:

$$
\boldsymbol{\sigma}(r)=\mathbf{C}: \boldsymbol{\varepsilon}^{e}(r),
$$

where $\mathbf{C}$ denotes the elastic moduli with classic symmetry properties: $C_{i j k l}=C_{j i k l}=C_{i j k k}=C_{k l i j}$.

The Helmholtz free energy $\phi$ per unit volume, for a system with volume $V$, under isotherm and quasistatic conditions, is assumed here to be only equal to the elastic energy $W_{e}=\frac{1}{2} \boldsymbol{\sigma}:\left(\boldsymbol{\varepsilon}-\boldsymbol{\varepsilon}^{p}\right)$ per unit volume:

$$
\phi=\frac{1}{V} \int_{V} W_{e} d V
$$

Then, $\phi$ reduces to:

$$
\phi=\frac{1}{2 V} \int_{V} \sigma_{i j}(r) \varepsilon_{i j}(r) d V-\frac{1}{2 V} \int_{V} \sigma_{i j}(r) \varepsilon_{i j}^{p}(r) d V .
$$

So $\phi$ depends on the boundary conditions and on the incompatible field $\boldsymbol{\varepsilon}^{p}(r)$.

Under the assumption of macro-homogeneous conditions, the overall strain and stress are respectively given by $\mathbf{E}=\frac{1}{V} \int_{V} \boldsymbol{\varepsilon}(r) d V$ and $\boldsymbol{\Sigma}=\frac{1}{V} \int_{V} \boldsymbol{\sigma}(r) d V$. Because of homogeneous elasticity, $\mathbf{E}=\mathbf{E}^{e}+\mathbf{E}^{p}$, where $\mathbf{E}^{e}=\frac{1}{V} \int_{V} \boldsymbol{\varepsilon}^{e}(r) d V$ and $\mathbf{E}^{p}=\frac{1}{V} \int_{V} \boldsymbol{\varepsilon}^{p}(r) d V$ are respectively the overall elastic and plastic strains. Furthermore, Eq. (5) can be simplified by using integrations by parts and the macroscopic Hooke's law given by:

$$
\Sigma_{i j}=C_{i j k} E_{k l}^{e} .
$$


This leads to:

$$
\phi\left(E_{i j}, \varepsilon_{i j}^{p}\right)=\frac{1}{2} C_{i j k l}\left(E_{k l}-E_{k l}^{p}\right) E_{i j}-\frac{1}{2 V} \int_{V} \sigma_{i j}(r) \varepsilon_{i j}^{p}(r) d V .
$$

The Legendre transform of the Helmholtz free energy gives the Gibbs free energy per unit volume:

$$
\psi\left(\Sigma_{i j}, \varepsilon_{i j}^{p}\right)=-\phi\left(E_{i j}, \varepsilon_{i j}^{p}\right)+\Sigma_{i j} E_{i j}=\frac{1}{2} \Sigma_{i j} E_{i j}+\frac{1}{2 V} \int_{V} \sigma_{i j}(r) \varepsilon_{i j}^{p}(r) d V .
$$

By using the macroscopic relation $E_{i j}=S_{i j k l} \Sigma_{k l}+E_{i j}^{p}$ in Eq. (8), we get:

$$
\psi\left(\Sigma_{i j}, \varepsilon_{i j}^{p}\right)=\frac{1}{2} \Sigma_{i j} S_{i j k l} \Sigma_{k l}+\frac{1}{2} \Sigma_{i j} E_{i j}^{p}+\frac{1}{2 V} \int_{V} \sigma_{i j}(r) \varepsilon_{i j}^{p}(r) d V,
$$

where $\mathbf{S}=\mathbf{C}^{-1}$ denotes the elastic compliances. Now, we assume that the local stress $\boldsymbol{\sigma}(r)$ can be expressed through the macroscopic stress $\Sigma$ and a fluctuation $\tau(r)$ corresponding to the internal stresses such that:

$$
\sigma_{i j}(r)=\Sigma_{i j}+\tau_{i j}(r) .
$$

Hence, we deduce from Eq. (9):

$$
\psi\left(\Sigma_{i j}, \varepsilon_{i j}^{p}\right)=\frac{1}{2} \Sigma_{i j} S_{i j k l} \Sigma_{k l}+\Sigma_{i j} E_{i j}^{p}+\frac{1}{2 V} \int_{V} \tau_{i j}(r) \varepsilon_{i j}^{p}(r) d V .
$$

According to this last equation, the Gibbs free energy per unit volume depends on the boundary conditions and on the prescribed plastic strain field $\boldsymbol{\varepsilon}^{p}(r)$ through $\mathbf{E}^{p}$ and through a volume integral containing internal stresses $\tau(r)$. For an infinite medium, the field equations can be solved using the Green's function technique through the modified Green's tensor $\Gamma\left(r-r^{\prime}\right)$ introduced by Kröner (1989) so that the strain field reads (Berveiller et al., 1987):

$$
\varepsilon_{i j}(r)=E_{i j}+\int_{V} \Gamma_{i j k l}\left(r-r^{\prime}\right) C_{k l p q}\left(\varepsilon_{p q}^{p}\left(r^{\prime}\right)-E_{p q}^{p}\right) d V^{\prime},
$$

where $\boldsymbol{\Gamma}\left(r-r^{\prime}\right)$ is deduced from the ordinary symmetric Green's tensor $\mathbf{G}(r)$ by:

$$
\Gamma_{i j k l}\left(r-r^{\prime}\right)=-\frac{1}{2}\left(G_{i k, j l}\left(r-r^{\prime}\right)+G_{j k, i l}\left(r-r^{\prime}\right)\right) .
$$

According to Kröner (1989), this last tensor can be expressed through a local part $\Gamma_{i j k l}^{l}\left(r-r^{\prime}\right)$ and a non-local one $\Gamma_{i j k l}^{n l}\left(r-r^{\prime}\right)$ scaling with $\frac{1}{\left\|r-r^{\prime}\right\|^{3}}$ such that:

$$
\Gamma_{i j k l}\left(r-r^{\prime}\right)=\Gamma_{i j k l}^{l}\left(r-r^{\prime}\right) \delta\left(r-r^{\prime}\right)+\Gamma_{i j k l}^{n l}\left(r-r^{\prime}\right) .
$$

In Eq. (13), $\mathbf{G}(r)$ solves the Navier equation:

$$
\left\{\begin{array}{l}
C_{p q k l} G_{k i, l q}\left(r-r^{\prime}\right)+\delta_{i p} \delta\left(r-r^{\prime}\right)=0 \\
\lim _{\left\|r-r^{\prime}\right\| \rightarrow \infty} G_{k m}\left(r-r^{\prime}\right)=0
\end{array}\right.
$$

Introducing Eq. (12) in the local Hooke's law $\boldsymbol{\sigma}(r)=\mathbf{C}:\left(\boldsymbol{\varepsilon}(r)-\boldsymbol{\varepsilon}^{p}(r)\right)$ yields:

$$
\sigma_{i j}(r)=-C_{i j k l}\left(\varepsilon_{k l}^{p}(r)-E_{k l}\right)+C_{i j k l} \int_{V} \Gamma_{k l m n}\left(r-r^{\prime}\right) C_{m n p q}\left(\varepsilon_{p q}^{p}\left(r^{\prime}\right)-E_{p q}^{p}\right) d V^{\prime} .
$$

Then, we introduce the macroscopic Hooke's law (Eq. (6)) in Eq. (10), so that:

$$
\tau_{i j}(r)=\sigma_{i j}(r)-C_{i j k l} E_{k l}^{e} .
$$

Thus, using Eq. (16) in the last equation yields:

$$
\tau_{i j}(r)=-C_{i j k l}\left(\varepsilon_{k l}^{p}(r)-E_{k l}^{p}\right)+C_{i j k l} \int_{V} \Gamma_{k l m n}\left(r-r^{\prime}\right) C_{m n p q}\left(\varepsilon_{p q}^{p}\left(r^{\prime}\right)-E_{p q}^{p}\right) d V^{\prime} .
$$

Hence

$$
\tau_{i j}(r)=\int_{V} l_{i j p q}\left(r-r^{\prime}\right)\left(\varepsilon_{p q}^{p}\left(r^{\prime}\right)-E_{p q}^{p}\right) d V^{\prime},
$$

where

$$
l_{i j p q}\left(r-r^{\prime}\right)=-C_{i j p q} \delta\left(r-r^{\prime}\right)+C_{i j k l} \Gamma_{k l m n}\left(r-r^{\prime}\right) C_{m n p q} .
$$

From Eq. (19), it is easy to determine the internal stress field generated by plastic fields.

Replacing $\tau(r)$ in Eq. (11) by Eq. (19) gives the expression for the Gibbs free energy per unit volume as a function of $\Sigma$ and $\boldsymbol{\varepsilon}^{p}(r)$ : 


$$
\psi\left(\Sigma_{i j}, \varepsilon_{i j}^{p}\right)=\frac{1}{2} \sum_{i j} S_{i j k l} \Sigma_{k l}+\Sigma_{i j} E_{i j}^{p}+\frac{1}{2 V} \int_{V}\left[\int_{V} l_{i j p q}\left(r-r^{\prime}\right)\left(\varepsilon_{p q}^{p}\left(r^{\prime}\right)-E_{p q}^{p}\right) d V^{\prime}\right] \varepsilon_{i j}^{p}(r) d V .
$$

Both Eqs. (19) and (21) indicate the complexity of interactions between plastic heterogeneities.

\subsection{Modeling slip bands by periodic oblate spheroids constrained by a spherical grain boundary}

Now, we consider that plastic deformation is distributed along slip bands inside an individual grain. Due to curved grain boundaries, we assume that the slip bands are described by oblate spheroids (Fig. 2) which are considered periodic, with a characteristic period $h$, inside a spherical grain of radius $R$ and volume $V_{g}$ (Fig. 1 ). Moreover, the use of oblate spheroids allows to take advantage of the Eshelby's properties for ellipsoidal inclusions (Eshelby, 1957). We use the following terminology: the length of the largest half axis of oblate spheroid (denoted by 'a' in Fig. 2) is called the oblate radius or the slip band radius, and the length of the smallest half axis of oblate spheroid (denoted by ' $c$ ' in Fig. 2) is called the oblate thickness or the slip band thickness for the sake of simplicity.

We consider a single slip system with unit vector $\mathbf{m}$ in the slip direction and unit vector $\mathbf{n}$ normal to the slip plane. Thus, this static configuration implies that all the slip bands $I$ with volume $V_{I}$ are coplanar and elongated in the slip plane with the smallest half axis $c$ in the direction of $\mathbf{n}$.

Hence, two major non-dimensional internal length scale parameters inherent to the microstructure are introduced. The first one is $\frac{h}{R}$ which characterizes the spatial distribution of slip bands. In this study, these ones are considered equally spaced for the sake of simplicity. The second one is $\frac{c}{R}$ which dictates the morphology of slip bands, i.e. their aspect ratio. As a consequence, the volume fraction of slip bands inside the grain depends on both parameters.

The associated plastic strain is defined through the Schmid tensor $R_{i j}=\frac{1}{2}\left(m_{i} n_{j}+m_{j} n_{i}\right)$ and the slip $\gamma(r)$ by:

$$
\varepsilon_{i j}^{p}(r)=R_{i j} \gamma(r)
$$

where

$$
\gamma(r)=\sum_{I} \gamma_{I} \theta_{I}(r)= \begin{cases}\gamma_{I} & \text { if } r \in V_{I} \\ 0 & \text { if } r \notin V_{I}\end{cases}
$$

and where $\theta_{I}(r)$ is the characteristic function. It depends on the location of the slip band $I$, on the volume $V_{I}$, and, on the fact that the bands are constrained by the grain boundary. Hence, Eq. (22) reads:

$$
\varepsilon_{i j}^{p}(r)=\sum_{I} \varepsilon_{i j}^{p_{I}} \theta_{I}(r)
$$

where $\varepsilon_{i j}^{p_{I}}=R_{i j} \gamma_{I}$ is the plastic strain in the Ith slip band. So, the average plastic strain over the grain is:

$$
\overline{\varepsilon_{i j}^{p}}=\sum_{I} f^{I} \varepsilon_{i j}^{p_{I}}
$$

where $f^{I}=\frac{V_{I}}{V_{g}}$ is the volume fraction of the Ith slip band in the grain.

The macroscopic strain is deduced from Eq. (24) by:

$$
E_{i j}^{p}=\frac{1}{V} \int_{V} \varepsilon_{i j}^{p}(r) d V=f^{g} \sum_{I} f^{I} \varepsilon_{i j}^{p_{I}}=f^{g} \bar{\varepsilon}_{i j}^{p g},
$$

where $f^{g}=\frac{V_{g}}{V}$ is the grain volume fraction. Since the plastic strain is concentrated in a grain of volume $V_{g}$ embedded in an infinite volume $V$, we get $f^{g} \ll 1$. Thus, we can neglect $E_{i j}^{p}$ with respect to $\varepsilon_{i j}^{p_{I}}$ for the computation of internal stresses in Eqs. (19) and (21).

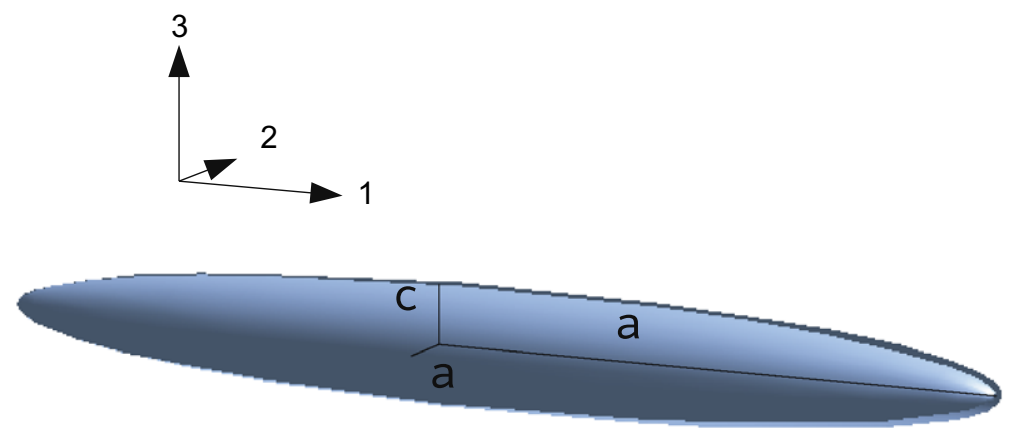

Fig. 2. A slip band is represented by an oblate spheroid with half axes $(a, a, c)$. 
Now, if we use Eq. (24) in Eq. (19), the internal stresses simplify into:

$$
\tau_{i j}(r)=\sum_{I} l_{i j p q}^{l}(r) \varepsilon_{p q}^{p_{1}}
$$

with

$$
l_{i j p q}^{I}(r)=-C_{i j p q} \theta^{I}(r)+C_{i j k l}\left(\int_{V_{I}} \Gamma_{k l m n}\left(r-r^{\prime}\right) d V^{\prime}\right) C_{m n p q} .
$$

Then, the average internal stress over a given slip band $N$ is:

$$
\bar{\tau}_{i j}^{N}=\frac{1}{V_{N}} \int_{V_{N}} \tau_{i j}(r) d V=\sum_{I} L_{i j p q}^{N I} \varepsilon_{p q}^{p_{I}},
$$

with $\mathbf{L}^{N I}=\frac{1}{V_{N}} \int_{V_{N}} \mathbf{I}^{I}(r) d V$. For convenience, we define the interaction tensor of oblate spheroids $I$ over $N$ by:

$$
T_{k l m n}^{N I}=\frac{1}{V_{N}} \int_{V_{N}} \int_{V_{l}} \Gamma_{k l m n}\left(r-r^{\prime}\right) d V^{\prime} d V
$$

In the particular case where $I=N, \mathbf{T}^{N N}$ is related to the elastic Eshelby's tensor $\mathbf{S}^{e s h, N}$ by:

$$
S_{k l p q}^{e s h N}=T_{k l m n}^{N N} C_{m n p q} .
$$

The expression of $\mathbf{L}^{N I}$ then simplifies to:

$$
\begin{aligned}
& L_{i j p q}^{N N}=-C_{i j k l}\left(I_{k l p q}-S_{k l p q}^{e s h N}\right) \\
& L_{i j p q}^{N I}=C_{i j k l} T_{k l m n}^{N I} C_{m n p q}, \text { if } N \neq I .
\end{aligned}
$$

Consequently, we get:

$$
\bar{\tau}_{i j}^{N}=-C_{i j k l}\left[I_{k l p q}-S_{k l p q}^{e s h, N}\right] \varepsilon_{p q}^{p_{N}}+\sum_{\substack{I \\ I \neq N}} C_{i j k l} T_{k l m n}^{N I} C_{m n p q} \varepsilon_{p q}^{p_{I}} .
$$

Now, using Eqs. (24) and (26) in Eq. (21), we deduce the expression of the Gibbs free energy per unit volume:

$$
\psi\left(\Sigma_{i j}, \varepsilon_{i j}^{p}\right)=\frac{1}{2} \sum_{i j} S_{i j k l} \Sigma_{k l}+f^{g} \sum_{i j} \bar{\varepsilon}_{i j}^{\bar{p} g}+W^{\text {internal }},
$$

where $W^{\text {internal }}$ is the internal part of $\psi\left(\Sigma_{i j}, \varepsilon_{i j}^{p}\right)$ :

$$
W^{\text {internal }}=\frac{1}{2} f^{g} \sum_{N} \sum_{I} f^{N} \varepsilon_{i j}^{p_{N}} L_{i j p q}^{N I} \varepsilon_{p q}^{p_{I}} .
$$

Actually, $W^{\text {internal }}$ can be decomposed into a first part due to the self-energies of slip bands $W^{\text {self }}$, and, a second part due to interaction energies between the different slip bands $W^{\text {inter }}$ such that $W^{\text {internal }}=W^{\text {self }}+W^{\text {inter }}$. The expression of $W^{\text {self }}$ reads:

$$
W^{\text {self }}=\frac{1}{2} f^{g} \sum_{N} f^{N} \varepsilon_{i j}^{p_{N}} L_{i j p q}^{N N} \varepsilon_{p q}^{p_{N}},
$$

and, the expression of $W^{\text {inter }}$ between the different slip bands $I$ and $N(I \neq N)$ is:

$$
W^{\text {inter }}=\frac{1}{2} f^{g} \sum_{N} \sum_{\substack{I \\ I \neq N}} f^{N} \varepsilon_{i j}^{p_{N}} L_{i j p q}^{N I} \varepsilon_{p q}^{p_{I}} .
$$

It is noteworthy that the expression for the average internal stress over a given slip band $N$ (Eq. (29)) can be retrieved from the Gibbs free energy per unit volume (Eqs. (35) and (36)) by computing the driving force associated with the plastification process $\left(\varepsilon_{i j}^{p_{N}}\right)$ inside the band $N$ denoted $F_{i j}^{N}$ (e.g., see Rice (1975); Maugin and Muschik (1994)). It writes $F_{i j}^{N}=\frac{\partial \psi_{N}}{\partial \varepsilon_{i j}^{p_{N}}}$ so that $\bar{\tau}_{i j}^{N}=\frac{1}{f^{g} f^{N}} F_{i j}^{N}-\Sigma_{i j}$.

For comparison, let us consider now the application of the classic Eshelby's method to the spherical grain of radius $R$ embedded in an infinite medium of volume $V$. Furthermore, a single slip system is considered like previously and characterized by the unit vector normal to the slip plane $\mathbf{n}$ and the unit vector along the slip direction $\mathbf{m}$. Then, the plastic strain components are uniform in the grain and are defined as:

$$
\varepsilon_{i j}^{p}(r)= \begin{cases}\varepsilon_{i j}^{p_{0}} & \text { if }\|r\| \leqslant R \\ 0 & \text { if }\|r\|>R\end{cases}
$$


where $\varepsilon_{i j}^{p_{0}}=R_{i j} \gamma$, and $\gamma$ constitutes a uniform plastic shear produced by slip bands inside the grain. Thus, the internal stresses for interior points to the grain, denoted $\tau_{i j}^{0}$, are uniform and depend on $\varepsilon_{i j}^{p_{0}}$ and elastic properties. It comes directly from Eshelby (1957) (considering $f^{g} \ll 1$ ):

$$
\tau_{i j}^{0}=-C_{i j k l}\left[I_{k l p q}-S_{k l p q}^{e s h}\right] \varepsilon_{p q}^{p_{0}}
$$

where in the particular case of a spherical grain and isotropic elastic properties:

$$
S_{k k k k}^{e s h}=\frac{7-5 v}{15(1-v)}, \quad S_{\substack{k k p p \\ k \neq p}}^{e s h}=\frac{5 v-1}{15(1-v)}, \quad \underset{\substack{k p k p \\ k \neq p}}{e s h}=\frac{4-5 v}{15(1-v)},
$$

where $v$ is the elastic Poisson's ratio.

In the Eshelby's uniform plastic grain representation, the internal part of the Gibbs free energy per unit volume simply yields according to Mura (1987):

$$
W^{\text {internal0 }}=-\frac{1}{2} f^{g} \varepsilon_{i j}^{p_{0}} C_{i j k l}\left(I_{k l p q}-S_{k l p q}^{e s h}\right) \varepsilon_{p q}^{p_{0}} .
$$

Let us remark that Eqs. (40) and (42) can be respectively deduced from Eqs. (34) and (37) considering the whole grain as a single spherical plastic inclusion.

In order to exhibit the internal length scale effects of the microstructure at a given macroscopic stress, we will compare the results at same $\mathbf{E}^{p}$, so that the Gibbs free energy only depends on the variations of the internal energy $W^{\text {internal }}$ (see Eq. (35)).

\subsection{Application to an in situ grain with periodic slip band distribution}

From the configuration depicted in Fig. 1, we consider a spherical grain with a periodic distribution of slip bands characterized in the coordinates of the active slip system by the unit vector in slip direction $\mathbf{m}=(0,1,0)$ and the unit vector normal to the slip plane $\mathbf{n}=(0,0,1)$. Thus, the Schmid tensor $R_{i j}$ introduced in Section 2.2 has only two non-zero components: $R_{23}=R_{32}=\frac{1}{2}$. So, the plastic strain in the Ith slip band $\varepsilon^{p_{1}}$ reduces to two non-zero components $\varepsilon_{23}^{p_{1}}=\varepsilon_{32}^{p_{1}}=\frac{1}{2} \gamma_{1}$. Then, both non-zero components for $\boldsymbol{\varepsilon}^{p}(\boldsymbol{r})$ and $\mathbf{E}^{p}$ are the $\{(2,3) ;(3,2)\}$ components. We deduce from Eq. (24):

$$
\varepsilon_{23}^{p}(r)=\varepsilon_{32}^{p}(r)=\sum_{I} \varepsilon_{23}^{p_{I}} \theta_{I}(r)
$$

and, from Eq. (26):

$$
E_{23}^{p}=E_{32}^{p}=f^{g} \sum_{I} f^{I} \varepsilon_{23}^{p_{I}} .
$$

We consider isotropic elastic properties characterized by Lamé elastic moduli (shear modulus $\mu$ and $\lambda$ ) so that $C_{i j k l}=\lambda \delta_{i j} \delta_{k l}+\mu\left(\delta_{i k} \delta_{j l}+\delta_{i l} \delta_{j k}\right)$.

For any $(i, j)$, Eq. (29) simplifies to:

$$
\bar{\tau}_{i j}^{N}=2 \sum_{I} L_{i j 23}^{N I} \varepsilon_{23}^{p_{I}} .
$$

When $(i, j) \notin\{(2,3) ;(3,2)\}, L_{i j 23}^{N I}$ is deduced from Eqs. (32) and (33) as follows:

$$
\begin{aligned}
& L_{i j 23}^{N N}=\lambda \delta_{i j} S_{p p 23}^{e s h}+2 \mu S_{i j 23}^{e s h, N} \\
& L_{i j 23}^{N I}=\lambda \mu \delta_{i j}\left(T_{p p 23}^{N I}+T_{p p 32}^{N I}\right)+2 \mu^{2}\left(T_{i j 23}^{N I}+T_{i j 32}^{N I}\right), \text { if } N \neq I .
\end{aligned}
$$

We do not give more details on $\bar{\tau}_{i j}^{N}$ since only the components $\{(2,3) ;(3,2)\}$ are needed to compute the resolved shear stress in Section 3.1. (see Eq. (58)). These ones are obtained from Eq. (45) with:

$$
\begin{aligned}
& L_{2323}^{N N}=L_{3223}^{N N}=-2 \mu\left(\frac{1}{2}-S_{2323}^{e s h, N}\right) \\
& L_{2323}^{N I}=L_{3223}^{N I}=2 \mu^{2}\left(T_{2323}^{N I}+T_{2332}^{N I}\right), \text { if } N \neq I,
\end{aligned}
$$

deduced from Eqs. (32) and (33).

Now using Eqs. (43) and (44) in Eq. (35), we deduce the expression of the Gibbs free energy per unit volume:

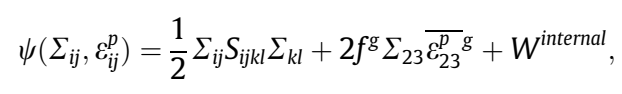

with according to Eq. (36): 


$$
W^{\text {internal }}=2 f^{g} \sum_{N} \sum_{I} f^{N} \varepsilon_{23}^{p_{N}} L_{2323}^{N I} \varepsilon_{23}^{p_{I}}
$$

Like in Section 2.2, we can write $W^{\text {internal }}=W^{\text {self }}+W^{\text {inter }}$ where $W^{\text {self }}$ and $W^{\text {inter }}$ are obtained using respectively Eqs. (37) and (38):

$$
W^{\text {self }}=2 f^{g} \sum_{N} f^{N} L_{2323}^{N N}\left(\varepsilon_{23}^{p_{N}}\right)^{2}
$$

and

$$
W^{i n t e r}=2 f^{g} \sum_{\substack{I \\ I \neq N}} f^{N} \varepsilon_{23}^{p_{N}} L_{2323}^{N I} \varepsilon_{23}^{p_{I}} .
$$

The expression of $S_{2323}^{e s h}$ for oblate spheroids is recalled in Appendix A, and, the expressions of $T_{2323}^{N I}$ and $T_{2332}^{N I}$ are computed in Appendix B. Let us remark that only these components are needed for the calculations in the case of an in situ grain as reported in this section as well as for polycrystalline aggregates in Section 3.

For comparison, we now consider the application of the classic Eshelby's method with $\mathbf{n}=(0,0,1)$ and $\mathbf{m}=(0,1,0)$. Then, the only non-zero plastic strain components are like before shear components $\varepsilon_{23}^{p}(r)=\varepsilon_{32}^{p}(r)$ defined as:

$$
\varepsilon_{23}^{p}(r)= \begin{cases}\varepsilon_{23}^{p_{0}} & \text { if }\|r\| \leqslant R \\ 0 & \text { if }\|r\|>R\end{cases}
$$

where $\varepsilon_{23}^{p_{0}}=\frac{1}{2} \gamma$. In this mean field approximation, $\gamma$ constitutes a uniform plastic shear produced by slip bands inside the grain. The internal stress for interior points to the grain $\tau_{23}^{0}$ comes directly from Eq. (40):

$$
\tau_{23}^{0}=-2 \mu \frac{7-5 v}{15(1-v)} \varepsilon_{23}^{p_{0}} .
$$

The internal part of the Gibbs free energy per unit volume simply yields according to Eq. (42):

$$
W^{\text {internal0 }}=\frac{1}{V} 8 \mu \pi R^{3} \frac{7-5 v}{45(v-1)}\left(\varepsilon_{23}^{p_{0}}\right)^{2} .
$$

In Fig. 3, we report the internal part of the Gibbs free energy per unit volume ( $W^{\text {internal }}$ in Eq. (50)) as a function of the number of slip bands. It means that different ratios $\frac{h}{R}$ are examined assuming that the slip bands are equally spaced and covers the whole grain for a fixed $\frac{c}{R}$. In order to simplify the comparison with the Eshelby's solution, we normalize the energies with respect to $\frac{8 \pi \mu R^{3}\left(\overline{\varepsilon_{23}^{p}}\right)^{2}}{V}$, where $\overline{\varepsilon_{23}^{p}}$ is the volume average of $\varepsilon_{23}^{p}(r)$ in the grain as defined by Eq. (25) and taken equal to the plastic strain $\varepsilon_{23}^{p 0}$ introduced in the uniform plastic Eshelby's inclusion (Eq. (54)). The normalized internal part of the Gibbs free energy per unit volume for the Eshelby's solution associated with a spherical inclusion gives the constant value $\frac{7-5 v}{45(v-1)}$ accord-

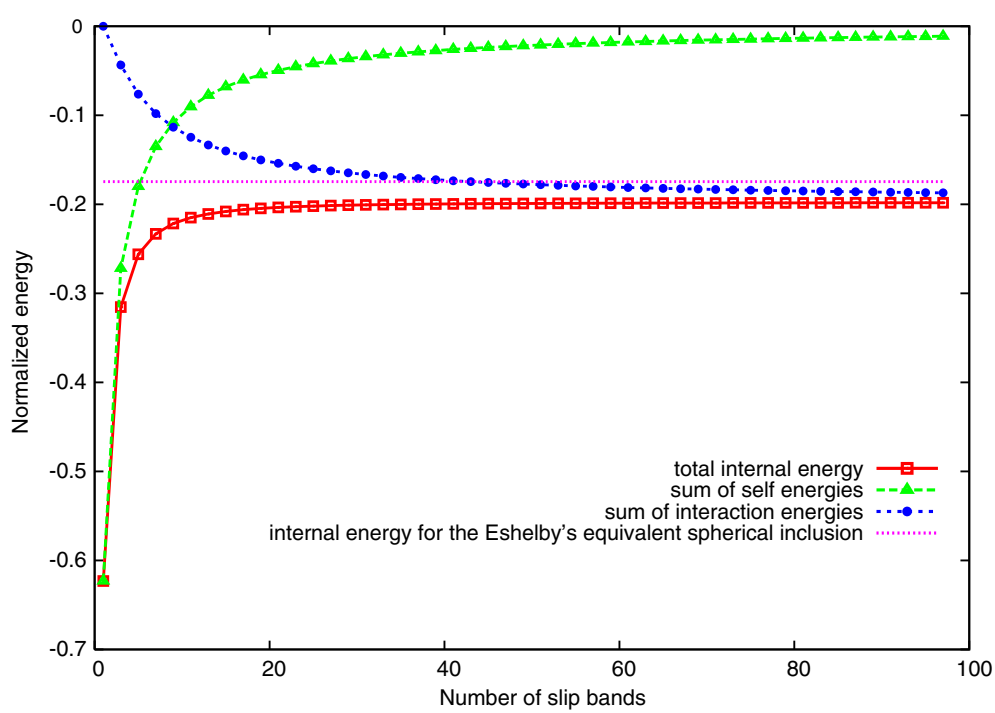

Fig. 3. Normalized energies: total internal energy (squares), sum of self-energies (triangles), sum of interaction energies (circles) as a function of the number of slip bands (modeled by oblate spheroids as described in Fig. 1 ) in a spherical grain $(R=1 \mu \mathrm{m})$. The slip bands have constant thickness such that $\frac{c}{R}=0.01$, and, the Poisson's ratio is set to $v=0.3$. Comparison with the Eshelby's solution (dashed lines). 
ing to Eq. (56). We also report the normalized parts due to self (Eq. (52)) and interaction (Eq. (53)) energies as a function of the number of slip bands to determine their respective influence on the total internal energy.

In these simulations, we chose $v=0.3$. Furthermore, the grain radius is set arbitrarily to $R=1 \mu \mathrm{m}$ and the thickness of slip bands is chosen constant such that $\frac{c}{R}=0.01$. In order to compare the normalized internal part of the Gibbs free energy per unit volume (denoted $W_{N}^{\text {internal }}$ ) for the periodic distribution of slip bands with the Eshelby's solution, the mean slip over the grain is kept constant and set to $\bar{\gamma}^{g}=2 \bar{\varepsilon}_{23}^{p}=0.01$.

We can notice that increasing the number of slip bands in the grain results in increasing the normalized internal Gibbs energy which gets closer and closer to the Eshelby's solution but does not converge towards it (Fig. 3). Let us note that using thinner slip bands (i.e. lower values of $\frac{c}{R}$ ) does not ensure the convergence towards the Eshelby's result as well. The main reason is that the plastified volume of slip bands cannot fill up the whole grain, but only up to about $65 \%$ of the grain volume. Thus, the contribution of the purely elastic regions between the slip bands still plays a role which explains the existing difference with the Eshelby's result in the asymptotic behavior (i.e. for the largest possible number of slip bands). This result is different from the one obtained by considering periodic distributions of glide loops (Berbenni et al., 2008) where increasing the number of loops inside the grain leads to the convergence towards the Eshelby's solution. This is essentially due to the possibility to fill up the whole grain by the loops in the physical limit of mutual dislocation annihilation processes. Fig. 3 also shows that decreasing the number of slip bands reduces the interaction energies but also increases the contribution of selfenergies, because the concentration of slip in each band is higher to maintain a constant average slip over the grain (low volume fraction of slip bands). Conversely, when slip bands are numerous, the respective role of these energies switches.

In the following, we explore the influence of the spatial period $h$ between slip bands on $W_{N}^{\text {internal }}$ for different numbers of bands (from 3 to 99) and for a given grain radius $R$. Fig. 4 represents $W_{N}^{\text {internal }}$ as a function of the non-dimensional internal length scale parameter $\frac{h}{R}$ when $\frac{c}{R}$ is set to 0.01 . We observe that for a given number of slip bands, $W_{N}^{\text {internal }}$ reaches a maximum for a critical value of $\frac{h}{R}$. Furthermore, as the number of bands is increased, this critical value decreases (but does not scale linearly with $\frac{h}{R}$ ), and, the corresponding maximum value for $W_{N}^{\text {internal }}$ increases without reaching the Eshelby's solution. These simulations show that for a given number of equally-spaced bands in a grain, we can deduce an optimized configuration defined by a critical value of $\frac{h}{R}$ denoted by $\frac{h_{\text {opt }}}{R}$ and reported in Table 1 . The existence of a maximum for $W_{N}^{\text {internal }}$ depends on both the spatial distribution and the morphology of slip bands which act simultaneously in a complex way. For high $\frac{h}{R}$ values with respect to $\frac{h_{\text {opt }}}{R}$ and in the case of a few slip bands, these ones are located far from each other, and, their morphologies are much different from the middle to the top of the grain. Thus, the part of $W_{N}^{\text {internal }}$ due to self-energies is mainly responsible for low levels of $W_{N}^{\text {internal }}$. Conversely, for low $\frac{h}{R}$ values with respect to $\frac{h_{\text {opt }}}{R}$, the slip bands are close to each other and their volume fraction in the grain becomes important which leads to low local slip in each band. In this case, the part of $W_{N}^{\text {internal }}$ due to interaction energies is predominant and monitors a decrease of $W_{N}^{\text {internal }}$ for low $\frac{h}{R}$. Finally, between these two states (low and high $\frac{h}{R}$ ), the opposite contributions of the two parts due to self- and interaction-energies make occur a configuration of slip bands for which $W_{N}^{\text {internal }}$ is maximum. The influence of the second non-dimensional internal length scale parameter $\frac{c}{R}$ is also explored in Fig. 5. The number of slip bands is limited to nine because large values of $\frac{c}{R}$ (up to 0.1) prevent from having more than nine bands inside the grain. For the same number of slip bands spreading into the grain, we found that the larger $\frac{c}{R}$, the higher $W_{N}^{\text {internal }}$. In addition, the corresponding values of $\frac{h_{\text {opt }}}{R}$ are increased as $\frac{c}{R}$ increases (Table 1 ). Moreover, the effect of $\frac{c}{R}$ becomes less important as the number of slip bands increases. Indeed, in this case, the part of $W_{N}^{\text {internal }}$ due to interaction energies also

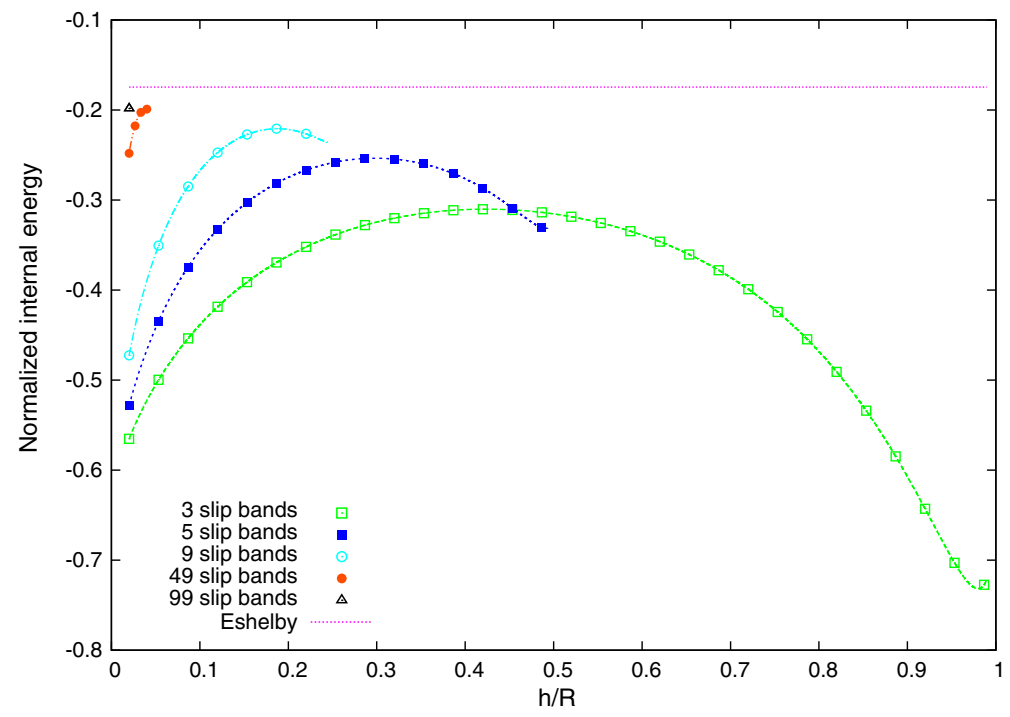

Fig. 4. Normalized internal part of the Gibbs free energy per unit volume (denoted $W_{N}^{\text {internal }}$ in the text) as a function of non-dimensional internal length parameter $\frac{h}{R}$ for various numbers of slip bands in the grain. Comparison with the Eshelby's solution (dashed lines). 
Table 1

Numerical values for $\frac{h_{\text {opt }}}{R}$ at different $\frac{c}{R}$ for numbers of slip bands inside the grain ranging from 3 to 99

\begin{tabular}{|c|c|c|c|}
\hline$\frac{c}{R}$ & 0.001 & 0.01 & 0.1 \\
\hline 3 bands & 0.419 & 0.422 & 0.460 \\
\hline 5 bands & 0.296 & 0.298 & 0.322 \\
\hline 9 bands & 0.186 & 0.187 & 0.200 \\
\hline 49 bands & 0.039 & 0.039 & 1 \\
\hline 99 bands & 0.020 & 0.020 & i \\
\hline
\end{tabular}

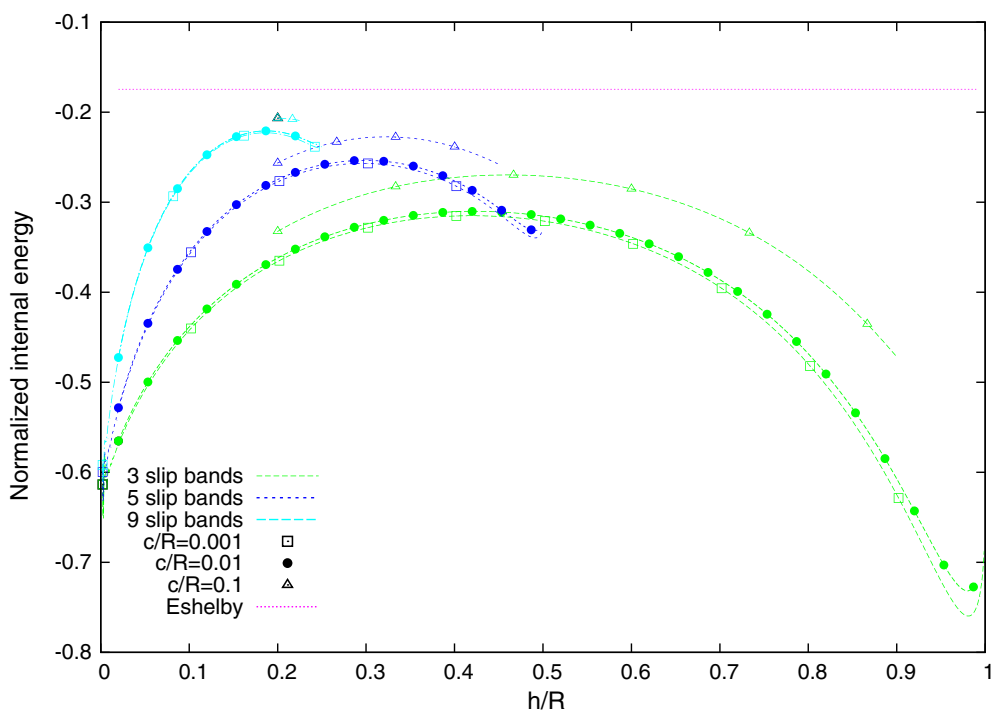

Fig. 5. Normalized internal part of the Gibbs free energy per unit volume (denoted $W_{N}^{\text {internal }}$ in the text) as a function of $\frac{h}{R}$ for various numbers of slip bands in the grain and for three different values of $\frac{c}{R}$ (square: 0.001 ; circles: 0.01 ; triangles: 0.1 ). Comparison with the Eshelby's solution (dashed lines).

become predominant (see Fig. 3), and $\frac{c}{R}$ has less influence on the part due to interaction energies than on the one due to selfenergies. From Fig. 5, the coupled effects of both non-dimensional internal length scale parameters are more pronounced for a small number of slip bands.

\section{Polycrystalline aggregates}

In this section, we focus on the plastic behavior of polycrystalline metals assuming intra-granular single slip concentrated into slip bands as described in Section 2. The Representative Volume Element (RVE) of the polycrystalline aggregate is constituted of a large number of spherical grains. In order to simplify the description of the aggregate, we assume that all the grains have a uniform size. First, we describe the constitutive equations for the single crystal behavior using rate dependent crystal plasticity. Secondly, the scale transition rule to obtain the polycrystalline aggregate behavior is presented. Finally, the numerical results on the overall plastic behavior as a function of the grain size are sketched.

\subsection{Single crystal behavior}

In a first attempt, we assume that in each grain $g$ of the polycrystal, a single slip system is able to be active during the whole plastification process. At the first loading step, we select the slip system having the largest resolved shear stress $\bar{\tau}_{r}^{g}$ for all possible slip systems $s(g)$ defined by $\bar{\tau}_{r}^{g}=\operatorname{Max}_{s(g)}\left(R_{i j}^{s(g)} \bar{\sigma}_{i j}{ }^{g}\right)$, where $\overline{\boldsymbol{\sigma}}^{g}$ is the stress due to the concentration over the grain of the applied stress. This last one is obtained by a scale transition rule as the one detailed in Section 3.2 . Each $\mathbf{R}^{s(g)}$ denotes the Schmid tensor of the slip system $s(g)$. The average stress in the Ith slip band of the grain $g$ is then given by $\overline{\boldsymbol{\sigma}}^{I(g)}=\overline{\boldsymbol{\sigma}}^{g}+\overline{\boldsymbol{\tau}}^{I(g)}$, where $\bar{\tau}^{I(g)}$ is the intra-granular average internal stress on $I(g)$ as calculated by Eq. (29). Then, during plastification, the evolution of the resolved shear stress in each slip band is given by:

$$
\bar{\tau}_{r}^{I(g)}=R_{i j}^{g} \bar{\sigma}_{i j}^{I(g)}=R_{i j}^{g}\left(\bar{\sigma}_{i j}^{g}+\bar{\tau}_{i j}^{I(g)}\right),
$$

where $\mathbf{R}^{g}$ is the Schmid tensor associated to the active slip system. This equation simplifies into the active slip system coordinates. Let $\mathbf{Q}^{g}$ be the rotation matrix from the active slip system coordinates to the crystal coordinates. For example, if we 
consider a slip direction with unit vector $\mathbf{m}=(0,1,0)$ and a unit vector normal to the slip plane $\mathbf{n}=(0,0,1)$, we get

$$
\begin{gathered}
\widetilde{\mathbf{R}}^{g}={ }^{t} \mathbf{Q}^{g} \cdot \mathbf{R}^{g} \cdot \mathbf{Q}^{g}=\left(\begin{array}{ccc}
0 & 0 & 0 \\
0 & 0 & \frac{1}{2} \\
0 & \frac{1}{2} & 0
\end{array}\right) \text { and then } \\
\bar{\tau}_{r}^{I(g)}=\widetilde{R}_{i j}^{g}\left(\overline{\tilde{\sigma}}_{i j}^{g}+\overline{\tilde{\tau}}_{i j}^{I(g)}\right)=\overline{\tilde{\sigma}}_{23}^{g}+\overline{\tilde{\tau}}_{23}^{I(g)},
\end{gathered}
$$

where $\overline{\tilde{\boldsymbol{\sigma}}}^{g}={ }^{t} \mathbf{Q}^{g} \cdot \overline{\boldsymbol{\sigma}}^{g} \cdot \mathbf{Q}^{g}, \overline{\tilde{\tau}}^{I(g)}={ }^{t} \mathbf{Q}^{g} \cdot \bar{\tau}^{I(g)} \cdot \mathbf{Q}^{g}$, and $\overline{\tilde{\tau}}_{23}^{I(g)}$ is computed using Eqs. (45), (48) and (49) applied to this example. Furthermore, the average resolved shear stress on the slip bands is then given by:

$$
\bar{\tau}_{r}^{b(g)}=\frac{1}{f^{p(g)}} \sum_{I(g)} f^{I(g)} \bar{\tau}_{r}^{I(g)},
$$

where $f^{p(g)}=\sum_{I(g)} f^{I(g)}$ is the total plastic volume fraction in the grain $g$.

In order to determine the evolution of plastic strain inside grains, we assume that slip rates in each slip band $I(g)$ follow a viscoplastic power law (e.g., see Pan and Rice (1983); Asaro and Needleman (1985)):

$$
\dot{\gamma}_{I(g)}=\dot{\gamma}^{0}\left(\frac{\left|\bar{\tau}_{r}^{I(g)}\right|}{\tau_{c}^{l(g)}}\right)^{n} \operatorname{sign}\left(\bar{\tau}_{r}^{I(g)}\right),
$$

where the exponent $n$ and $\dot{\gamma}^{0}$ are the same for all grains. The exponent $n$ characterizes the strain rate sensitivity of the material (generally, $n$ is relatively high for metals) and $\dot{\gamma}^{0}$ is a reference slip rate. Besides, $\tau_{c}^{l(g)}$ is a reference resolved shear stress in each slip band $I(g)$. For the sake of simplicity, we will consider it as constant and the same for all grains such that $\tau_{c}^{l(g)}=\tau_{c}^{0}$, even though it may depend on the evolution of the slip $\gamma_{I(g)}$ due to strain-hardening mechanisms in slip bands (not detailed here). Then, we can compute in each band $I(g)$ the plastic slip $\gamma_{I(g)}(t+\Delta t)$ through a time integration scheme. So we get the average slip in the plastic bands $\bar{\gamma}^{b(g)}=\frac{1}{f p(g)} \sum_{I(g)} f^{I(g)} \gamma_{I(g)}$ and in the grain $\bar{\gamma}^{g}=\sum_{I(g)} f^{I(g)} \gamma_{I(g)}$.

Finally, we can compute the average plastic strain for the grain in the crystal coordinates as follows:

$$
\bar{\varepsilon}_{i j}^{p(g)}=R_{i j}^{g} \bar{\gamma}^{g} .
$$

\subsection{Scale transition from single crystal to polycrystal}

To model the polycrystal's behavior, we first consider a Representative Volume Element (RVE) constituted of $N$ grains with different crystallographic orientations. It is submitted to a macroscopic homogeneous stress denoted by $\widehat{\boldsymbol{\Sigma}}$ in the global coordinates. For each grain $g$ with a given crystallographic orientation, we define by $\mathbf{P}^{g}$ the rotation matrix from the crystal coordinates to the global coordinates. So we get $\boldsymbol{\Sigma}^{g}={ }^{t} \mathbf{P}^{g} \cdot \widehat{\boldsymbol{\Sigma}} \cdot \mathbf{P}^{g}$. Now, for each grain $g$, we perform the calculations in the crystal coordinates. We express the average local stress in the grain by using the diluted Eshelby approximation for the sake of simplicity, so that the interaction law (for homogeneous elasticity) yields:

$$
\bar{\sigma}_{i j}^{g}=\sum_{i j}^{g}-C_{i j k l}\left(I_{k l p q}-S_{k l p q}^{e s h}\right) \bar{\varepsilon}_{p q}^{p(g)},
$$

where $\overline{\boldsymbol{g}}^{p(g)}$ is the average plastic strain on the grain and $\mathbf{S}^{\text {esh }}$ is the elastic Eshelby tensor for an ellipsoidal shaped grain (Eshelby, 1957).

Since we have calculated the average plastic strain $\overline{\boldsymbol{g}}^{p(g)}$ in each grain $g$ of the polycrystal in the previous section, we can express it in the global coordinates $\overline{\hat{\boldsymbol{\varepsilon}}}^{p(g)}=\mathbf{P}^{g} \cdot \overline{\boldsymbol{\varepsilon}}^{p(g)} \cdot{ }^{t} \mathbf{P}^{g}$, and the overall plastic strain can thus be deduced, from the average relation:

$$
\widehat{\mathbf{E}}^{p}=\sum_{g} f_{g} \overline{\hat{\boldsymbol{\varepsilon}}}^{p(g)},
$$

where $f_{g}$ are the grain volume fractions. Thus, the overall total strain $\widehat{\mathbf{E}}$ reads:

$$
\widehat{E}_{i j}=\widehat{E}_{i j}^{e}+\widehat{E}_{i j}^{p}=\widehat{S}_{i j k l} \widehat{\Sigma}_{k l}+\widehat{E}_{i j}^{p} \text {. }
$$

Let us remark that even though the interaction law based on the diluted Eshelby's model is known to give a too stiff estimation of the overall response and is only valid for very low level of plastification (generally the average plastic volume fraction in the whole RVE does not exceed 15\%), the present objective is to study qualitatively the impact of internal length parameters on the macroscopic strain hardening. We do not focus on the accurate description of inter-granular interactions.

\subsection{Results and discussion on polycrystalline aggregates}

In this section, we consider a RVE constituted of 100 spherical grains with random crystallographic orientations. As an illustration, we choose isotropic elastic constants. Young modulus $E$ and Poisson's ratio $v$ are reported in Table 2. In 
Table 2

Materials parameters introduced for the simulations of the initial plastic stage of deformation for nickel

\begin{tabular}{llllll}
\hline Parameters & $E(\mathrm{GPa})$ & $v$ & $\dot{\gamma}^{0}\left(\mathrm{~s}^{-1}\right)$ & $n$ & $\tau_{c}^{0}(\mathrm{MPa})$ \\
\hline Values & 214 & 0.3 & $10^{-2}$ & 100 & 5 \\
\hline
\end{tabular}

Eq. (60), we set the different materials parameters $\dot{\gamma}^{0}, n$ and $\tau_{c}^{0}$ to realistic values for fcc metals, as given in Table 2 . In order to simplify the description of the aggregate, we assume that the grain size distribution is uniform. For all the grains, the intra-granular microstructure (periodic distribution of slip bands) is characterized by a single slip system with a given spatial period and same numbers of bands. For each number of slip bands, and for a given $\frac{c}{R^{\prime}}$, we use the values of $\frac{h_{\text {opt }}}{R}$, obtained in Section 2.3 through Figs. 4 and 5, which maximize the internal part of the Gibbs free energy.

We report in Fig. 6 the influence of $\frac{h_{\text {opt }}}{R}$ on the overall tensile stress-strain response with $\frac{c}{R}$ set to 0.01 . First, we observe that the polycrystal's hardening is stronger for large $\frac{h_{o p t}}{R}$, namely when the plastic deformation is concentrated in a few slip bands. Indeed, there is an important decrease of the hardening rate from 1 to 3 slip bands. Second, we can notice that considering such periodic distributions of slip bands makes the associated tensile curve converge towards a limit one. For low $\frac{h_{o p t}}{R}$ values corresponding to high numbers of slip bands, the curves superimpose. In comparison, the tensile response obtained with the classic Eshelby's model for homogeneous plastic grains leads to a much lower hardening. This is due to the fact that in the mean field approach, only inter-granular accommodations operate whereas for the intra-granular microstructure described in Section 2, a new source of internal stresses occurs (see Eq. (57)). Furthermore, at the onset of plasticity, the overall plastic behavior is mainly dictated by the progressive activation of slip systems inside grains following the assumptions described in Section 3.1 (for each grain, the single slip system with the highest Schmid factor is activated). Then, after the very low strain regime (i.e. $E_{11}^{p}>10^{-7}$ ), the evolution of slip inside bands becomes significant as well as the effect of $\frac{h_{\text {opt }}}{R}$ on the overall strain-hardening. Fig. 7 represents the combined effects of $\frac{h_{\text {opt }}}{R}$ and $\frac{c}{R}$ on the overall stress-strain response of the polycrystal. We find that the larger $\frac{c}{R}$, the lower the macroscopic flow stress. This softening effect can be linked to the results obtained in Section 2.3 through Fig. 5 for large $\frac{c}{R}$. We also find that the effect of $\frac{c}{R}$ on the macroscopic flow stress is more pronounced in the case of 1 band. Conversely, this one gets lower as $\frac{h_{\text {opt }}}{R}$ decreases (Fig. 7). These results show that the strain-hardening of the polycrystal is strongly affected by the discrete nature of slip (through $\frac{h}{R}$ ) and by the morphology of slip bands (through $\frac{c}{R}$ ). When $\frac{h}{R}$ is low, the intra-granular discrete distribution of slip bands has less impact on the mechanical behavior (Fig. 6).

Experimental studies of slip band distributions during tensile tests have been recently reported in single crystals (Zaiser, 2006; Schwerdtfeger et al., 2007). Such issues still have to be investigated in the case of polycrystals due to a complex internal length scale effect associated with the presence of grain boundaries. Both experimental and theoretical works are now needed in order to relate in a more accurate manner slip line patterns with grain size and plastic deformation evolution. The current limitations of the present approach lie in: (i) the assumption of periodicity for the intra-granular distribution of slip bands, and, (ii) the relative simplicity of single slip configurations. However, our main objective clearly remains to break through the classic mean field Eshelby's approach by first assuming simple intra-granular configurations observed for slip bands.

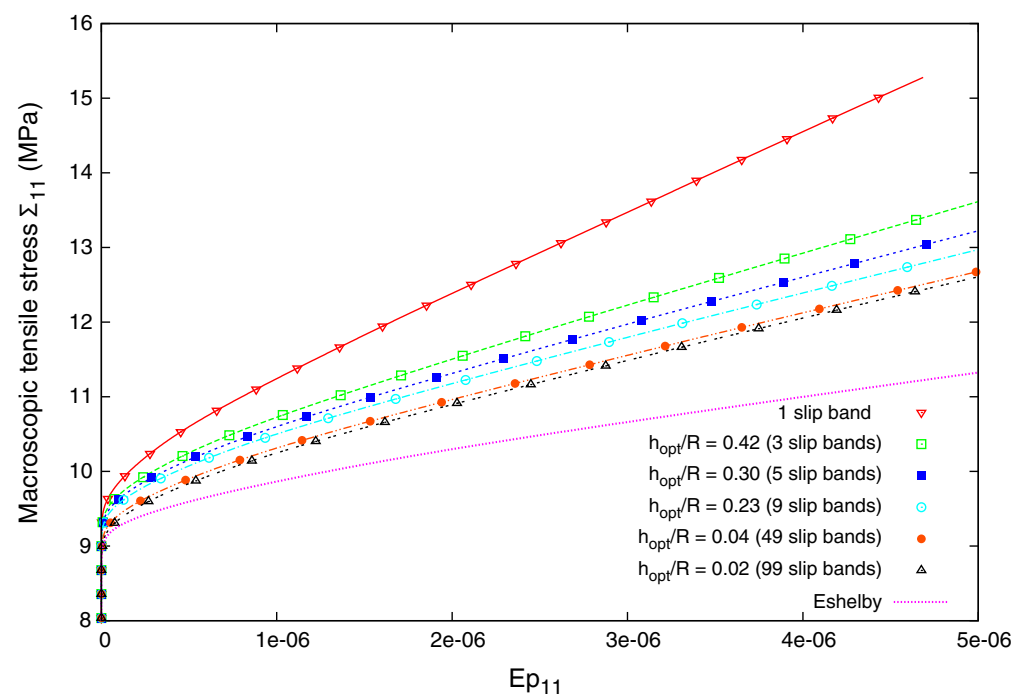

Fig. 6. Role of internal length scale parameter $\frac{h_{o p t}}{R}$ on the macroscopic tensile stress-strain responses (points) for a polycrystal constituted of 100 randomly oriented spherical grains with $\frac{c}{R}=0.01$. The particular case of 1 slip band is also reported (inverse triangles). Comparison with the stress-strain response given by the classic Eshelby's model (dashed line). 


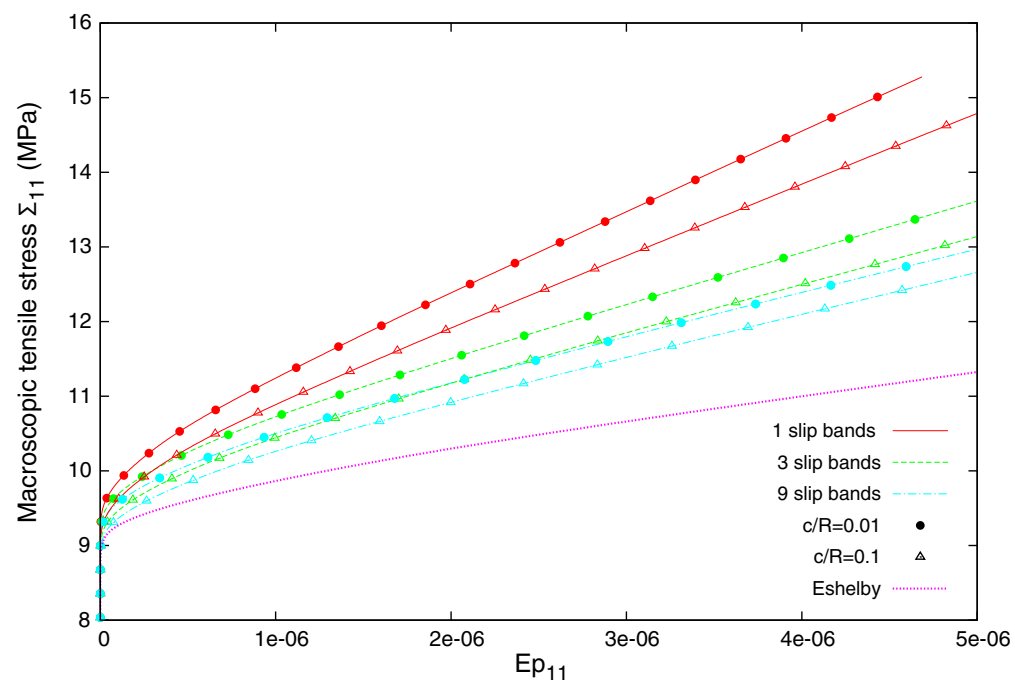

Fig. 7. Combined effects of internal length scale parameters $\frac{h_{\text {opt }}}{R}$ and $\frac{c}{R}$ on the macroscopic tensile stress-strain response for a polycrystal constituted of 100 randomly oriented spherical grains. The particular case of 1 slip band is also reported. Comparison with the stress-strain response given by the classic Eshelby's model (dashed line).

\section{Conclusions and perspectives}

Throughout this paper, intra-granular plastic slip heterogeneities such as slip bands occurring in the micro-plastic regime of polycrystalline metals are modeled by discrete periodic distributions of coplanar flat ellipsoidal plastic inclusions (oblate spheroids). First of all, we considered the field equations and the thermodynamics framework associated with an isolated in situ plastic grain embedded in an infinite elastic matrix. Two types of slip configurations are studied in the grain of spherical shape assuming single slip. The first type of configuration corresponds to the classic plastic Eshelby's inclusion where slip is supposed uniform over the grain. The second type of configurations takes into account intra-granular slip bands constrained by the spherical grain boundary and represented by a periodic distribution of coplanar oblate spheroids. Then, in addition to the grain size, the microstructure is characterized by the spatial period of slip bands (denoted $h$ ) and the thickness of the bands (denoted $c$ ). In Section 2, we investigated the role of both non-dimensional internal length scale parameters

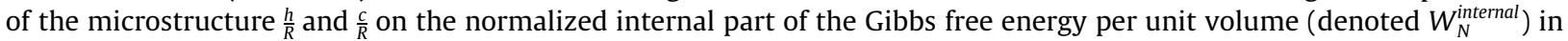
the case of an in situ grain. For a given number of slip bands inside the grain, we found there exists a critical value $\frac{h}{R}$ which maximizes $W_{N}^{\text {internal }}$. This critical value also depends on $\frac{c}{R}$. Moreover, the role of both internal length scale parameters on $W_{N}^{\text {internal }}$ is all the more pronounced as the slip is localized. In Section 3, the theory is applied to polycrystalline aggregates where low volume fractions of grains undergo plastification, i.e. at the onset of plasticity. In this case, the inter-granular accommodation rule is supposed to follow a simple diluted Eshelby's model and the two aforementioned intra-granular slip configurations are considered in each grain for comparisons. The Representative Volume Element of the polycrystal is given by a set of 100 random crystallographic orientations and the texture development is disregarded. In each grain, crystal plasticity is restricted to the most active slip system and two hypotheses are used for intra-granular slip as previously. It is either uniform (classic Eshelby's mean field approach) or localized in periodic slip bands represented by coplanar oblate spheroids. We found that both aforementioned non-dimensional internal parameters have strong influences on the flow stress of polycrystals when the spatial period of slip bands is on the same order as the grain radius. For such slip band configurations, an increase of $\frac{h}{R}$, or, a decrease of $\frac{c}{R}$, leads to an increase of the polycrystal's strain-hardening. For a given metallic polycrystal, the knowledge of slip band spatial distribution and slip band heights will be the next steps to investigate in order to improve the description of intra-granular slip.

\section{Acknowledgements}

The authors acknowledge the French ANR for financial support under research contract LIOM. The simulations were performed with the scientific computing code Simula+ (LPMM/LAMAV).

\section{Appendix A. Eshelby's tensor for an oblate spheroid}

In the present study, intra-granular slip bands are modeled by oblate spheroids with half axes $(a, a, c)$ with $a>c$. Their volume is given by $V_{\text {oblate }}=\frac{4}{3} \pi a^{2} c$. 
In this case, the Eshelby tensor reads (Eshelby, 1957; Mura, 1987):

$$
S_{2323}^{e s h}=\frac{a^{2}+c^{2}}{16 \pi(1-v)} I_{23}+\frac{1-2 v}{16 \pi(1-v)}\left(I_{2}+I_{3}\right),
$$

where

$$
\begin{aligned}
& I_{2}=\frac{2 \pi \zeta}{\left(1-\zeta^{2}\right)^{3 / 2}}\left[\arccos (\zeta)-\zeta \sqrt{1-\zeta^{2}}\right], \\
& I_{3}=4 \pi-2 I_{2}, \\
& I_{23}=\frac{I_{2}-I_{3}}{c^{2}-a^{2}}=\frac{3 I_{2}-4 \pi}{a^{2}\left(\zeta^{2}-1\right)} .
\end{aligned}
$$

with $\zeta=\frac{c}{a}$. Hence, if we introduce these last expressions in Eq. (A.1), we obtain:

$$
\begin{aligned}
S_{2323}^{\text {esh }} & =\frac{1}{16 \pi(1-v)}\left[\frac{1+\zeta^{2}}{1-\zeta^{2}}\left(4 \pi-3 I_{2}\right)+(1-2 v)\left(4 \pi-I_{2}\right)\right], \\
& \left.\left.=\frac{1}{16 \pi(1-v)}\left[\frac{1+\zeta^{2}}{1-\zeta^{2}}+(1-2 v)\right) 4 \pi-3 \frac{1+\zeta^{2}}{1-\zeta^{2}}+(1-2 v)\right) I_{2}\right] \\
& \left.\left.=\frac{1}{4(1-v)}\left[2\left(\frac{1}{1-\zeta^{2}}-v\right)-\frac{2+\zeta^{2}}{1-\zeta^{2}}-v\right) \frac{\zeta}{\left(1-\zeta^{2}\right)} \frac{\arccos (\zeta)}{\sqrt{1-\zeta^{2}}}-\zeta\right)\right] .
\end{aligned}
$$

\section{Appendix B. Pair interaction tensor for two coplanar oblate spheroids}

The pair interaction tensor between two coplanar ellipsoidal inclusions (same directions for half axes), namely $V_{I}$ and $V_{J}$, as depicted in Fig. B.1 is defined by:

$$
t_{k l m j}^{I J}=-\frac{1}{V_{I}} \int_{V_{I}} \int_{V_{J}} G_{k m, l j}\left(r-r^{\prime}\right) d V^{\prime} d V
$$

where $\mathbf{G}$ denotes the Green tensor associated to the homogeneous (infinite) medium of moduli $\mathbf{C}$ which satisfies the following equation:

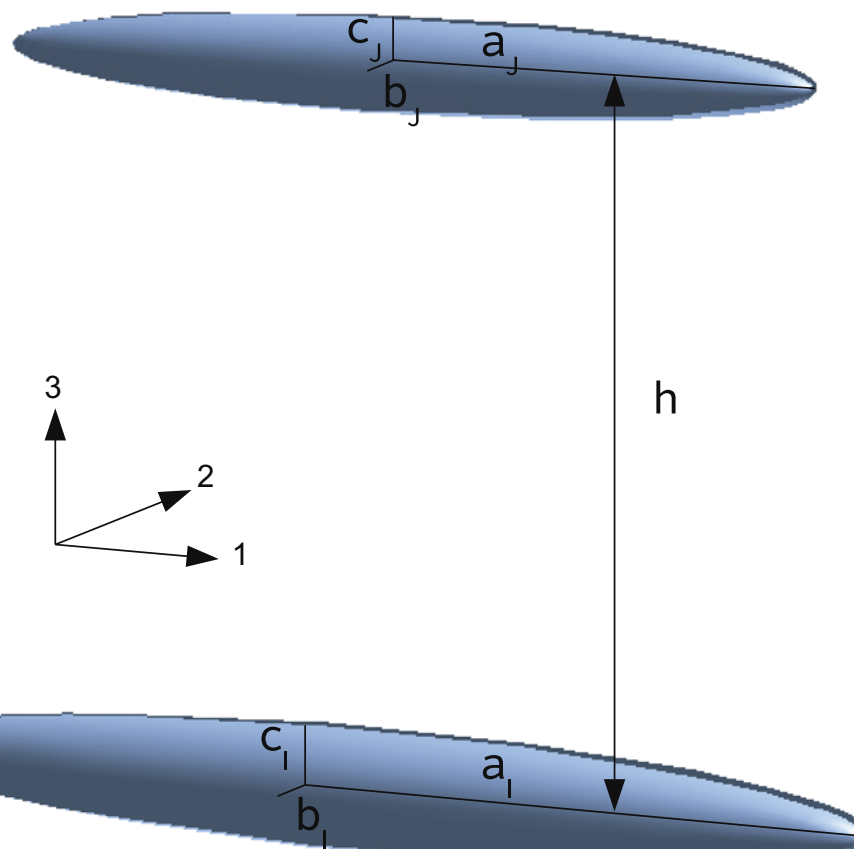

Fig. B.1. Interaction between two coplanar ellipsoidal inclusions of volume $V_{I}$ and $V_{J}$ with respective half axes $a_{I}, b_{I}, c_{I}$ and $a_{J}, b_{J}, c_{J}$. The separation distance between the two ellipsoid centers is denoted $h$ with $h>c_{I}+c_{J}$. 


$$
\left\{\begin{array}{l}
C_{i j k l} G_{k m, l j}\left(r-r^{\prime}\right)+\delta_{i m} \delta\left(r-r^{\prime}\right)=0 \\
\lim _{\left\|r-r^{\prime}\right\| \rightarrow \infty} G_{k m}\left(r-r^{\prime}\right)=0 .
\end{array}\right.
$$

The Green tensor $G_{k m}$ can be computed using the Fourier integral technique (Mura, 1987):

$$
G_{k m}\left(r-r^{\prime}\right)=\frac{1}{8 \pi^{3}} \int_{V_{\xi}} \widetilde{G_{k m}}(\xi) \mathrm{e}^{+\mathrm{i} \xi \cdot\left(\mathbf{r}-\mathbf{r}^{\prime}\right)} d V_{\xi},
$$

where $\xi$ is the vector of the Fourier space (i.e. conjugated of $\mathbf{r}$ ) with components $\left(\xi_{1}, \xi_{2}, \xi_{3}\right)$ and modulus $\|\xi\|=\sqrt{\xi_{1}^{2}+\xi_{2}^{2}+\xi_{3}^{2}}$. In Eq. (B.3), $\widehat{G_{k m}}(\xi)$ is the Fourier transform of $G_{k m}(r)$.

The Fourier transform of Eq. (B.2) yields:

$$
C_{i j k l} \widetilde{G_{k m}}(\xi) \xi_{l} \xi_{j}=\delta_{i m} .
$$

In the case of an homogeneous isotropic linear elastic medium as considered in the present study, $\mathbf{C}$ only depends on the Lamé constants, i.e. $\lambda$ and $\mu$ (shear modulus), so that $\widetilde{G_{k m}}(\xi)$ writes:

$$
\widetilde{G_{k m}}(\xi)=\frac{1}{\mu}\left(\frac{\delta_{k m}}{\xi^{2}}-\frac{\lambda+\mu}{\lambda+2 \mu} \frac{\xi_{k} \xi_{m}}{\xi^{4}}\right) \text {. }
$$

Following Berveiller et al. (1987), Eq. (B.3) can be used in Eq. (B.2):

$$
t_{k l m j}^{I J}=-\frac{1}{8 \pi^{3}} \frac{1}{V_{I}} \int_{V_{I}} \int_{V_{J}} \int_{V_{\xi}} \xi_{l} \xi_{j} \widetilde{G_{k m}}(\xi) \mathrm{e}^{+\mathrm{i} \xi \cdot\left(\mathbf{r}-\mathbf{r}^{\prime}\right)} d V_{\xi} d V^{\prime} d V .
$$

The mathematical integration can be performed applying the spherical coordinates such that $d V_{\xi}=\xi^{2} \sin \theta d \theta d \varphi d \xi$, and:

$$
\left\{\begin{array}{l}
\xi_{1}=\xi \sin \theta \cos \varphi=\xi \chi_{1}(\theta, \varphi), \\
\xi_{2}=\xi \sin \theta \sin \varphi=\xi \chi_{2}(\theta, \varphi), \\
\xi_{3}=\xi \cos \theta=\xi \chi_{3}(\theta),
\end{array}\right.
$$

where $\xi \in[0, \infty[, \theta \in[0, \pi]$, and, $\phi \in[0,2 \pi[$. After some derivations explicated in Berveiller et al. (1987), the following result is obtained for the pair interaction tensor $t_{k l m j}^{I j}$ in the peculiar case of two coplanar ellipsoidal inclusions (Fig. B.1) with respective half axes denoted $a_{I}, b_{I}, c_{I}$ and $a_{J}, b_{J}, c_{J}$ :

$$
t_{k l m j}^{I J}=\frac{1}{V_{I}} \frac{2}{\pi} a_{l} b_{I} c_{I} a_{J} b_{J} c_{J} \int_{0}^{2 \pi} \int_{0}^{\pi} \chi_{l}(\theta, \varphi) \chi_{j}(\theta, \varphi) \xi^{2} \widetilde{G_{k m}}(\xi) \frac{F(\theta, \varphi) \sin \theta}{\left[\left(a_{I}^{2} \chi_{1}^{2}+b_{I}^{2} \chi_{2}^{2}+c_{I}^{2} \chi_{3}^{2}\right)\left(a_{J}^{2} \chi_{1}^{2}+b_{J}^{2} \chi_{2}^{2}+c_{J}^{2} \chi_{3}^{2}\right)\right]^{\frac{3}{2}}} d \theta d \varphi .
$$

where $\xi^{2} \widetilde{G_{k m}}(\xi)$ only depends on elastic constants $\lambda, \mu$, and angles $\theta, \varphi$ as follows:

$$
\xi^{2} \widetilde{G_{k m}}(\xi)=\frac{1}{\mu}\left(\delta_{k m}-\frac{\lambda+\mu}{\lambda+2 \mu} \chi_{k}(\theta, \varphi) \chi_{m}(\theta, \varphi)\right) .
$$

The scalar function $F(\theta, \varphi)$ writes in this peculiar case following Berveiller et al. (1987):

$$
\begin{aligned}
F(\theta, \varphi)= & \frac{1}{24}\left(-\alpha^{3}+3 v \alpha^{2}+3 u \alpha^{2}-6 u v \alpha\right) \int_{0}^{+\infty} \frac{\sin (\alpha k)}{k} d k+\frac{1}{24}\left(\beta^{3}+3 v \beta^{2}-3 u \beta^{2}-6 u v \beta\right) \int_{0}^{+\infty} \frac{\sin (\beta k)}{k} d k \\
& +\frac{1}{24}\left(\gamma^{3}+3 v \gamma^{2}-3 u \gamma^{2}-6 u v \gamma\right) \int_{0}^{+\infty} \frac{\sin (\gamma k)}{k} d k+\frac{1}{24}\left(-\delta^{3}+3 v \delta^{2}+3 u \delta^{2}-6 u v \delta\right) \int_{0}^{+\infty} \frac{\sin (\delta k)}{k} d k
\end{aligned}
$$

with:

$$
\begin{aligned}
& \alpha=\alpha(\theta, \varphi)=u+v-w \\
& \beta=\beta(\theta, \varphi)=u-v-w \\
& \gamma=\gamma(\theta, \varphi)=u-v+w \\
& \delta=\delta(\theta, \varphi)=u+v+w
\end{aligned}
$$

and:

$$
\begin{aligned}
& u=u(\theta, \varphi)=\left(a_{I}^{2} \chi_{1}^{2}+b_{I}^{2} \chi_{2}^{2}+c_{I}^{2} \chi_{3}^{2}\right)^{\frac{1}{2}}, \\
& v=v(\theta, \varphi)=\left(a_{J}^{2} \chi_{1}^{2}+b_{J}^{2} \chi_{2}^{2}+c_{J}^{2} \chi_{3}^{2}\right)^{\frac{1}{2}}, \\
& w=w(\theta)=h \chi_{3} .
\end{aligned}
$$

The integrals in Eq. (B.10) are given by:

$$
\int_{0}^{+\infty} \frac{\sin (\alpha k)}{k} d k=\left\{\begin{array}{l}
+\frac{\pi}{2} \text { if } \alpha>0 \\
-\frac{\pi}{2} \text { if } \alpha<0
\end{array}\right.
$$


Following Berveiller et al. (1987), it is also noteworthy that:

$$
t_{k l m j}^{J I}=\frac{V_{I}}{V_{J}} t_{k l m j}^{I J}
$$

so that it is needed to compute $t_{k l m j}^{I J}$.

From this point, we apply the last expression for $t_{k l m j}^{I J}$ to the case of the interaction between two coplanar oblate spheroids. Thus, it is now assumed that $a_{I}=b_{I}$ and $a_{I}>c_{I}$ for $I$ (resp. $a_{J}=b_{J}$ and $a_{J}>c_{J}$ for $J$ ) which simplifies $t_{k l m j}^{I J}$. Actually, $F$ is now only dependent on $\theta$ which will simplify the numerical integration. Furthermore, using Eq. (B.9) into the last expression for $t_{k l m j}^{I J}$ yields:

$$
\begin{aligned}
t_{k l m j}^{I J}= & \frac{3}{2 \pi^{2}} \frac{1}{\mu} a_{J}^{2} c_{J} \\
& \times \int_{0}^{2 \pi} \int_{0}^{\pi} \chi_{l}(\theta, \varphi) \chi_{j}(\theta, \varphi)\left(\delta_{k m}-\frac{\lambda+\mu}{\lambda+2 \mu} \chi_{k}(\theta, \varphi) \chi_{m}(\theta, \varphi)\right) \frac{F(\theta) \sin \theta}{\left[\left(a_{I}^{2} \sin ^{2} \theta+c_{I}^{2} \cos ^{2} \theta\right)\left(a_{J}^{2} \sin ^{2} \theta+c_{J}^{2} \cos ^{2} \theta\right)\right]^{\frac{3}{2}}} d \theta d \varphi,
\end{aligned}
$$

where now $\alpha, \beta, \gamma, \delta$ (and so $F$ ) only depend on $\theta$ through $u(\theta), v(\theta)$ and $w(\theta)$ :

$$
\begin{aligned}
& u=\left(a_{I}^{2} \sin ^{2} \theta+c_{I}^{2} \cos ^{2} \theta\right)^{\frac{1}{2}}, \\
& v=\left(a_{J}^{2} \sin ^{2} \theta+c_{J}^{2} \cos ^{2} \theta\right)^{\frac{1}{2}}, \\
& w=h \cos \theta .
\end{aligned}
$$

In Section 2.3, it appears necessary to compute the symmetric part of $t_{k l m j}^{I J}$ denoted $T_{k l m j}^{I J}=\frac{1}{2}\left(t_{k l m j}^{I J}+t_{l k m j}^{I J}\right)$. Furthermore, only the components $T_{2332}^{I J}\left(=T_{3232}^{I J}\right)$ and $T_{2323}^{I J}\left(=T_{3223}^{I J}\right)$ are needed (see Section 2.3$)$. Thus, we only compute $t_{2332}^{I J}, t_{2323}^{I J}$ and $t_{3232}^{I J}$ since $t_{3223}^{I J}=t_{2332}^{I J}$ following the previous expression for $t_{k l m j}^{I J}$.

We first have:

$$
\begin{aligned}
t_{2332}^{I J} & =-\frac{3}{2 \pi^{2}} \frac{\lambda+\mu}{\mu(\lambda+2 \mu)} a_{J}^{2} c_{J} \int_{0}^{2 \pi} \int_{0}^{\pi} \chi_{2}^{2}(\theta, \varphi) \chi_{3}^{2}(\theta, \varphi) \frac{F(\theta) \sin \theta}{u^{3}(\theta) v^{3}(\theta)} d \theta d \varphi, \\
& =-\frac{3}{4 \pi^{2}} \frac{\lambda+\mu}{\mu(\lambda+2 \mu)} a_{J}^{2} c_{J} \int_{0}^{2 \pi}(1-\cos 2 \varphi) d \varphi \int_{0}^{\pi} \sin ^{2} \theta \cos ^{2} \theta \frac{F(\theta) \sin \theta}{u^{3}(\theta) v^{3}(\theta)} d \theta, \\
& =-\frac{3}{2 \pi} \frac{\lambda+\mu}{\mu(\lambda+2 \mu)} a_{J}^{2} c_{J} \int_{0}^{\pi} \frac{\sin ^{2} \theta \cos ^{2} \theta F(\theta) \sin \theta}{u^{3}(\theta) v^{3}(\theta)} d \theta,
\end{aligned}
$$

then, we get:

$$
\begin{aligned}
t_{2323}^{I J} & =\frac{3}{2 \pi^{2}} \frac{1}{\mu} a_{J}^{2} c_{J} \int_{0}^{2 \pi} \int_{0}^{\pi}\left[\chi_{3}^{2}(\theta, \varphi)\left(1-\frac{\lambda+\mu}{\lambda+2 \mu} \chi_{2}^{2}(\theta, \varphi)\right)\right] \frac{F(\theta) \sin \theta}{u^{3}(\theta) v^{3}(\theta)} d \theta d \varphi \\
& =\frac{3}{\pi} \frac{1}{\mu} a_{J}^{2} c_{J} \int_{0}^{\pi} \cos ^{2} \theta \frac{F(\theta) \sin \theta}{u^{3}(\theta) v^{3}(\theta)} d \theta+t_{2332}^{I} .
\end{aligned}
$$

and, afterwards:

$$
t_{3232}^{I J}=\frac{3}{2 \pi^{2}} \frac{1}{\mu} a_{J}^{2} c_{J} \int_{0}^{2 \pi} \sin ^{2} \varphi \int_{0}^{\pi} \sin ^{2} \theta \frac{F(\theta) \sin \theta}{u^{3}(\theta) v^{3}(\theta)} d \theta d \varphi+t_{2332}^{I J}=\frac{3}{2 \pi} \frac{1}{\mu} a_{J}^{2} c_{J} \int_{0}^{\pi} \sin ^{2} \theta \frac{F(\theta) \sin \theta}{u^{3}(\theta) v^{3}(\theta)} d \theta+t_{2332}^{I J} .
$$

It is noteworthy that the three integrals for $t_{2332}^{\prime \prime}, t_{2323}^{\prime \prime}$ and $t_{3232}^{\prime \prime}$ become single integrals. In order to compute these integrals, let us introduce the new variable $x=\cos \theta$, hence $d x=-\sin \theta d \theta$. Furthermore, we have:

$$
\begin{aligned}
& u=\left(a_{I}^{2}+\left(c_{I}^{2}-a_{I}^{2}\right) x^{2}\right)^{\frac{1}{2},} \\
& v=\left(a_{J}^{2}+\left(c_{J}^{2}-a_{J}^{2}\right) x^{2}\right)^{\frac{1}{2}}, \\
& w=h x .
\end{aligned}
$$

Thus, we can notice the following properties:

$$
\begin{aligned}
& \alpha(-x)=u(-x)+v(-x)-w(-x)=u(x)+v(x)+w(x)=\delta(x), \\
& \beta(-x)=u(-x)-v(-x)-w(-x)=u(x)-v(x)+w(x)=\gamma(x) .
\end{aligned}
$$


Hence, we deduce that $F(-x)=F(x)$, which allows us to write:

$$
\begin{aligned}
& t_{2332}^{I J}=\frac{3}{\pi} \frac{\lambda+\mu}{\mu(\lambda+2 \mu)} a_{J}^{2} c_{J} \int_{0}^{1} x^{2}\left(x^{2}-1\right) \frac{F(x)}{u^{3}(x) v^{3}(x)} d x, \\
& t_{2323}^{I J}=\frac{6}{\pi} \frac{1}{\mu} a_{J}^{2} c_{J} \int_{0}^{1} x^{2} \frac{F(x)}{u^{3}(x) v^{3}(x)} d x+t_{2332}^{I J}, \\
& t_{3232}^{I J}=\frac{3}{\pi} \frac{1}{\mu} a_{J}^{2} c_{J} \int_{0}^{1}\left(1-x^{2}\right) \frac{F(x)}{u^{3}(x) v^{3}(x)} d x+t_{2332}^{I J} .
\end{aligned}
$$

Finally, $T_{2332}^{I J}$ and $T_{2323}^{I J}$ can be computed as:

$$
T_{2332}^{I J}=\frac{1}{2}\left(t_{2332}^{I J}+t_{3232}^{I J}\right)=\frac{3}{\pi} \frac{1}{\mu} a_{J}^{2} c_{J} \int_{0}^{1}\left(x^{2}-1\right) \frac{F(x)}{u^{3}(x) v^{3}(x)}\left[\frac{\lambda+\mu}{\lambda+2 \mu} x^{2}-\frac{1}{2}\right] d x,
$$

and

$$
T_{2323}^{I J}=\frac{1}{2}\left(t_{2323}^{I J}+t_{3223}^{I J}\right)=\frac{3}{\pi} \frac{1}{\mu} a_{J}^{2} c_{J} \int_{0}^{1} x^{2} \frac{F(x)}{u^{3}(x) v^{3}(x)}\left[1+\frac{\lambda+\mu}{\lambda+2 \mu}\left(x^{2}-1\right)\right] d x,
$$

Then, the interaction term $T_{2323}^{I J}+T_{2332}^{I J}$ in Eq. (49) (i.e. for $I \neq J$ ) is derived as:

$$
T_{2323}^{I J}+T_{2332}^{I J}=\frac{6}{\pi} \frac{1}{\mu} a_{J}^{2} c_{J} \int_{0}^{1} \frac{F(x)}{u^{3}(x) v^{3}(x)}\left[\frac{\lambda+\mu}{\lambda+2 \mu} x^{2}\left(x^{2}-1\right)+\frac{1}{4}\left(x^{2}+1\right)\right] d x .
$$

Using Eq. (B.14) yields:

$$
T_{2323}^{J I}+T_{2332}^{I I}=\frac{V_{I}}{V_{J}}\left(T_{2323}^{I J}+T_{2332}^{I J}\right) .
$$

We can compute $T_{2323}^{I J}+T_{2332}^{I J}$ with a Gauss-Legendre numerical integration scheme (Press et al., 2002) since no analytical results is given in the literature from the author's knowledge in the case of two oblate spheroids (i.e. with $a_{I}>c_{I}$ ).

The CPU times to compute the integration points and weights are performed once and are reported in Table B.1. These CPU times are obtained on an intel Woodcrest 5160 ( $3 \mathrm{Ghz}, \times 86-64)$ using the intel C++ compiler.

In order to determine the needed effective number of integration points, we compute the numerical relative error defined by $\frac{\left|T_{n}-T_{n=10,00,00 \mid}\right|}{\left|T_{n=10,00,00}\right|}$ where $T_{n}=T_{2323}^{I J}+T_{2332}^{I J}$ for a given number of integration points $n$, and, where $T_{n=10,00,00}$ corresponds to $10,00,00$ integration points. The results are reported in Table B.2. They are obtained considering the most critical morphologies for oblate spheroids in the simulations corresponding to $\frac{c_{I}}{a_{I}} \approx 1 \times 10^{-3}$. We observe that a good accuracy is obtained from $n=20,000$ points which are used in all the simulations.

In order to check the relevancy of the numerical procedure, we compare the numerical results obtained for $T_{2323}^{I J}+T_{2332}^{I J}$ called $T_{n}$ to the analytical solution reported by Berveiller et al. (1987), called $T_{\text {analytical }}$, in the case of two spherical inclusions of volumes $V_{I}$ and $V_{J}$ with respective half axes $a_{I}=b_{I}=c_{I}$ and $a_{J}=b_{J}=c_{J}$. By denoting $k^{2}=\left(a_{I}^{2}+a_{J}^{2}\right) / h^{2}$ where $h$ is the sep-

\begin{tabular}{|c|c|c|c|c|c|c|}
\hline Integration points & 10 & 100 & 1000 & 10,000 & 20,000 & 50,000 \\
\hline CPU time (s) & $\sim 0$ & $\sim 0$ & 0.02 & 2.29 & 8.36 & 43.66 \\
\hline
\end{tabular}
aration distance between the centers of the two inclusions $\left(h>a_{I}+a_{J}\right)$, this solution writes (Berveiller et al., 1987):

$$
T_{2323}^{I J}+T_{2332}^{I J}=T_{\text {analytical }}=\frac{1}{6} \frac{a_{J}^{3}}{h^{3}} \frac{1}{\mu(1-v)}\left(1+v-\frac{12}{5} k^{2}\right) .
$$

The relative errors defined as $\frac{\left|T_{n}-T_{\text {analytical }}\right|}{\left|T_{\text {analytical }}\right|}$ are reported as a function of the number of integration points in Table B.3.

Table B.1

CPU time (in seconds) to compute the Gauss integration weights and points (performed once)

Table B.2

Relative errors for the numerical computation of $T_{2323}^{I J}+T_{2332}^{I J}$ in the case of two oblate spheroids with radii $a_{I}=a_{J}=100 \mu \mathrm{m}$ and $c_{I}=c_{J}=0.1 \mu \mathrm{m}$ distant from $h=2.7 \mu \mathrm{m}$ and $h=20 \mu \mathrm{m}$

\begin{tabular}{lllll}
\hline Integration points & 100 & 1000 & 10,000 & 50,000 \\
\hline Relative error $(h=20 \mu \mathrm{m})$ & $4.73 \times 10^{-2}$ & $6.06 \times 10^{-4}$ & $1.64 \times 10^{-6}$ & 20,000 \\
\hline Relative error $(h=2.7 \mu \mathrm{m})$ & 9.17 & 0.22 & $7.31 \times 10^{-5}$ & $4.83 \times 10^{-7}$ \\
\hline
\end{tabular}

Table B.3

Relative error for the numerical computation of $T_{2323}^{I J}+T_{2332}^{I J}$ in the case of two spherical inclusions with radii $a_{I}=0.1 \mu \mathrm{m}$ and $a_{J}=1 \mu \mathrm{m}$ distant from $h=2.7 \mu \mathrm{m}$

\begin{tabular}{lllll}
\hline Integration points & 100 & 1000 & 10,000 & 50,000 \\
\hline Relative error $(h=2.7 \mu \mathrm{m})$ & 0.12 & $1.07 \times 10^{-4}$ & $9.07 \times 10^{-6}$ & $3.64 \times 10^{-7}$ \\
\hline
\end{tabular}




\section{References}

Acharya, A., 2001. A model of crystal plasticity based on the theory of continuously distributed dislocations. Journal of the Mechanics and Physics of Solids 49, 761-785.

Acharya, A., Bassani, J.L., 2000. Lattice incompatibility and a gradient theory of crystal plasticity. Journal of the Mechanics and Physics of Solids 48 (8), $1565-$ 1595.

Acharya, A., Roy, A., 2006. Size effects and idealized dislocation microstructure at small scales: prediction of a phenomenological model of mesoscopic field dislocation mechanics: Part 1. Journal of the Mechanics and Physics of Solids 54, 1687-1710.

Aifantis, E.C., 1987. The physics of plastic deformation. International Journal of Plasticity 3, 211-247.

Aifantis, K.E., Willis, J.R., 2005. The role of interfaces in enhancing the yield strength of composites and polycrystals. Journal of the Mechanics and Physics of Solids 53, 1047-1070.

Armstrong, R.W., Codd, I., Douthwaite, M., Petch, N.J., 1962. The plastic deformation of polycrystalline aggregates. Philosophical Magazine 7, 45-58.

Asaro, R.J., Needleman, A., 1985. Texture development and strain hardening in rate dependent polycrystals. Acta Metallurgica 33, 923-953.

Balint, D.S., Deshpande, V.S., Needleman, A., der Giessen, E.V., 2008. Discrete dislocation plasticity analysis of the grain size dependence of the flow strength of polycrystals. International Journal of Plasticity 24 (12), 2149-2172.

Berbenni, S., Berveiller, M., Richeton, T., 2008. Intra-granular plastic slip heterogeneities: discrete vs. mean field approaches. International Journal of Solids and Structures 45, 4147-4172.

Berbenni, S., Favier, V., Berveiller, M., 2007. Impact of the grain size distribution on the behaviour of heterogeneous materials. International Journal of Plasticity 23, 114-142.

Berbenni, S., Favier, V., Lemoine, X., Berveiller, M., 2004. Micromechanical modeling of the elastic-viscoplastic behavior of polycrystalline steels having different microstructures. Materials Science and Engineering A 372, 128-136.

Berveiller, M., Fassi-Fehri, O., Hihi, A., 1987. The problem of two plastic and heterogeneous inclusions in an anisotropic medium. International Journal of Engineering Science 25 (6), 691-709.

Berveiller, M., Zaoui, A., 1979. An extension of the self-consistent scheme to plastically flowing polycrystals. Journal of the Mechanics and Physics of Solids $26,325-344$.

Cheong, K.S., Busso, E.P., Arsenlis, A., 2005. A study of microstructural length scale effects on the behavior of fcc polycrystals using strain gradient concepts. International Journal of Plasticity 21, 1797-1814.

Cleveringa, H., VanderGiessen, E., Needleman, A., 1998. Discrete dislocation simulations and size dependent hardening in single slip. Journal de Physique IV (France) 8. Pr4-83-92.

Eshelby, J.D., 1957. The determination of the elastic field of an ellipsoidal inclusion and related problems. Proceedings of the Royal Society London A 241 , 376-396.

Espinosa, H.D., Panico, M., Berbenni, S., Schwarz, K.W., 2005. Discrete dislocation dynamics simulations to interpret plasticity size and surface effects in freestanding fcc thin films. International Journal of Plasticity 22, 2091-2117.

Fleck, N.A., Hutchinson, J.W., 1997. Strain gradient plasticity. Advances in Applied Mechanics 33, 296-358.

Forest, S., Barbe, F., Cailletaud, G., 2000. Cosserat modelling of size effects in the mechanical behaviour of polycrystals and multi-phase materials. International Journal of Solids and Structures 37, 7105-7126.

Forest, S., Sedlacek, R., 2003. Plastic slip distribution in two-phase laminate microstructures: dislocation based versus generalized-continuum approaches. Philosophical Magazine 83 (2), 245-276.

Fréchard, S., Martin, F., Clément, C., Cousty, J., 2006. Afm and ebsd combined studies of plastic deformation in a duplex stainless steel. Materials Science and Engineering A 418, 312-319.

Gilormini, P., 1995. Insuffisance de l'extension classique du modele autocoherent au comportement non lineaire. Comptes Rendus de l'Academie des Sciences IIb 320, 115-122.

Groh, S., Devincre, B., Kubin, L.P., Roos, A., Feyel, F., Chaboche, J.L., 2005. Size effects in metal matrix composites. Materials Science and Engineering A 400$401,279-282$.

Groma, I., Csiksor, F.F., Zaiser, M., 2003. Spatial correlations and higher-order gradient terms in a continuum description of dislocation dynamics. Acta Materialia 51, 1271-1281.

Hill, R., 1965. Continuum micro-mechanics of elastoplastic polycrystals. Journal of the Mechanics and Physics of Solids 13, 89-101.

Hirth, J.P., 1972. The influence of grain boundaries on mechanical properties. Metallurgical Transactions 3, 3047-3067.

Hutchinson, J.W., 1976. Bounds and self-consistent estimates for creep of polycrystalline materials. Journal of the Mechanics and Physics of Solids 13, 89101.

Kröner, E., 1989. Modified green functions in the theory of heterogeneous and/or anisotropic linearly elastic media. In: Weng, G.J., Taya, M., Abe, M. (Eds.), Micromechanics and Inhomogeneity. Springer, Berlin, pp. 197-211.

Kubin, L.P., Canova, G., Condat, M., Devincre, B., Pontikis, V., Bréchet, Y., 1992. Dislocation microstructures and plastic flow: a 3d simulation. Solid State Phenomena 23-24, 455-472.

Lebensohn, R.A., Tomé, C.N., 1993. A self-consistent anisotropic approach for the simulation of plastic deformation and texture development of polycrystals: application to zirconium alloys. Acta Metallurgica and Materialia 41 (9), 2611-2624.

Lefebvre, S., Devincre, B., Hoc, T., 2007. Yield stress strengthening in ultrafine-grained metals: a two-dimensional simulation of dislocation dynamics. Journal of the Mechanics and Physics of Solids 55 (4), 788-802.

Li, J., Weng, G.J., 1997. A secant-viscosity approach to the time-dependent creep of an elastic-viscoplastic composite. Journal of the Mechanics and Physics of Solids 45, 1069-1083.

Margolin, H., Stanescu, M.S., 1975. Polycrystalline strengthening. Acta Metallurgica 23, 1411-1418.

Masson, R., Bornert, M., Suquet, P., Zaoui, A., 2000. An affine formulation for the prediction of the effective properties of non linear composites and polycrystals. Journal of the Mechanics and Physics of Solids 48 (6-7), 1203-1227.

Maugin, G.A., Muschik, W., 1994. Thermodynamics with internal variables: Part i. General concepts. Journal of Non-Equilibrium Thermodynamics 19, 217249.

Mercier, S., Molinari, A., 2009. Homogenization of elastic-viscoplastic heterogeneous materials: self-consistent and Mori-Tanaka schemes. International Journal of Plasticity 25, 1024-1048.

Molinari, A., 2002. Averaging models for heterogeneous viscoplastic and elastic-viscoplastic materials. Journal of Engineering Materials and Technology $124,62-70$.

Molinari, A., Canova, G.R., Ahzi, S., 1987. A self-consistent approach of the large deformation polycrystal viscoplasticity. Acta Metallurgica 35, $2983-2994$.

Mura, T., 1987. Micromechanics of Defects in Solids. Kluwer Academic Publishers., Dordrecht, The Netherlands.

Neuhäuser, H., 1983. Slip-line formation and collective dislocation motion. In: Nabarro, F.R.N. (Ed.), Dislocations in Solids, vol. 4. North-Holland, Amsterdam, pp. 319-440.

Pan, J., Rice, J.R., 1983. Rate sensitivity of plastic flow and implications for yield-surface vertices. International Journal of Solids and Structures 19 , 973-987.

Paquin, A., Berbenni, S., Favier, V., Lemoine, X., Berveiller, M., 2001. Micromechanical modeling of the elastic-viscoplastic behavior of polycrystalline steels. International Journal of Plasticity 17, 1267-1302.

Pierard, O., Doghri, I., 2006. An enhanced affine formulation and the corresponding numerical algorithms for the mean field homogenization of elastoviscoplastic composites. International Journal of Plasticity 22, 131-157. 
Press, W.H., Teukolsky, S.A., Vetterling, W.T., Flannery, B.P., 2002. Numerical recipes in C++. The art of scientific computing, second ed. Cambridge University Press, USA.

Rice, J.R., 1975. Continuum mechanics and thermodynamics plasticity in relation to microscale deformation mechanisms. In: Argon, A.S. (Ed.), Constitutive Equations in Plasticity. MIT Press, Cambridge, pp. 23-75.

Roy, A., Peerlings, R.H.J., Geers, M.G.D., Kasyanyuk, Y., 2008. Continuum modeling of dislocation interactions: why discreteness matters? Materials Science Engineering A 486, 653-661.

Sabar, H., Berveiller, M., Favier, V., Berbenni, S., 2002. A new class of micro-macro models for elastic-viscoplastic heterogeneous materials. International Journal of Solids and Structures 39, 3257-3276.

Schwarz, K.W., 1999. Simulation of dislocations on the mesoscopic scale I. Methods and examples. Journal of Applied Physics 85 (1), $108-119$.

Schwerdtfeger, J., Nadgorny, E., Madani-Grasset, F., Koutsos, V., Blackford, J.R., Zaiser, M., 2007. Scale-free statistics of plasticity-induced surface steps on kcl single crystals. Journal of Statistical Mechanics: Theory and Experiments 4, L04001.

VanderGiessen, E., Needleman, A., 1995. Discrete dislocation plasticity: a simple planar model. Modelling and Simutation in Materials Science Engineering 3, 689-735.

Verdier, M., Fivel, M., Groma, I., 1998. Mesoscopic scale simulation of dislocation dynamics in fcc metals: principles and applications. Modelling and Simulation in Materials Science Engineering 6, 755-770.

Villechaise, P., Sabatier, L., Girard, J., 2002. On slip band features and crack initiation in fatigued 316l austenitic stainless steel. Part 1: Analysis by electron back-scattered diffraction and atomic force microscopy. Materials Science and Engineering A 323, $377-385$.

Weng, G.J., 1980. Constitutive equations of single crystals and polycrystalline aggregates under cyclic loading. International Journal of Engineering Science 18, 1385-1397.

Zaiser, M., 2006. Scale invariance in plastic flow of crystalline solids. Advances in Physics 55 (1-2), 185-245.

Zaiser, M., Aifantis, E.C., 2006. Randomness and slip avalanches in gradient plasticity. International Journal of Plasticity $22,1432-1455$. 